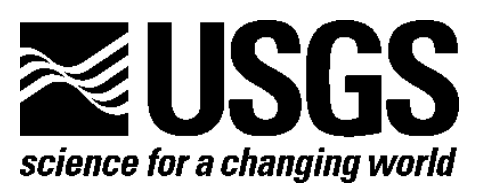

\title{
Legacy KIAr and ${ }^{40} \mathrm{Ar} / 3^{39} \mathrm{Ar}$ Geochronologic Data from the Alaska-Aleutian Range Batholith of South-central Alaska
}

By Lisa L. Koeneman and Frederic H. Wilson, compilers

Open-File Report 2018-1033

U.S. Department of the Interior

U.S. Geological Survey 


\section{U.S. Department of the Interior \\ RYAN K. ZINKE, Secretary}

\section{U.S. Geological Survey \\ William H. Werkheiser, Deputy Director \\ exercising the authority of the Director}

U.S. Geological Survey, Reston, Virginia: 2018

For more information on the USGS-the Federal source for science about the Earth,

its natural and living resources, natural hazards, and the environment-visit https://www.usgs.gov/ or call 1-888-ASK-USGS (1-888-275-8747).

For an overview of USGS information products, including maps, imagery, and publications, visit https://store.usgs.gov/.

Any use of trade, firm, or product names is for descriptive purposes only and does not imply endorsement by the U.S. Government.

Although this information product, for the most part, is in the public domain, it also may contain copyrighted materials as noted in the text. Permission to reproduce copyrighted items must be secured from the copyright owner.

Suggested citation:

Koeneman, L.L., and Wilson, F.H., comps., 2018, Legacy K/Ar and ${ }^{40} \mathrm{Ar} /{ }^{39} \mathrm{Ar}$ geochronologic data from the AlaskaAleutian Range batholith of south-central Alaska: U.S. Geological Survey Open-File Report 2018-1033, 8 p., 1 plate, https://doi.org/10.3133/ofr20181033.

ISSN 2331-1258 (online) 


\section{Contents}

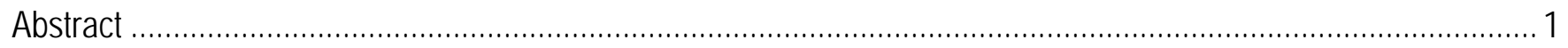

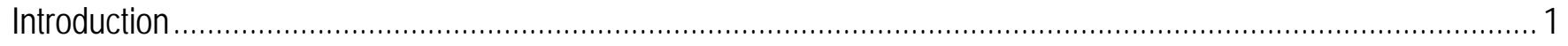

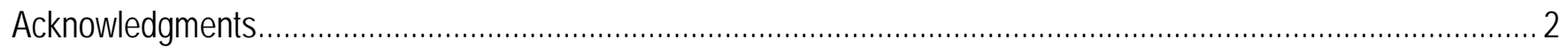

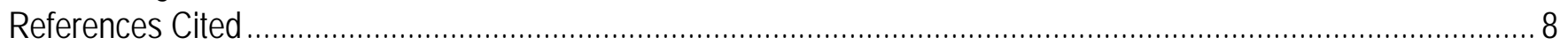

\section{Tables}

1. Potassium-argon analytical data and descriptive information for samples of the Alaska-Aleutian Range batholith in the Tyonek, Lime Hills, Kenai, Lake Clark, and Iliamna 1:250,000-scale quadrangles, south-central Alaska............................................................................................................... 2

2. Descriptive information and ${ }^{40} \mathrm{Ar} /{ }^{39} \mathrm{Ar}$ analytical data for samples of the Alaska-Aleutian Range batholith in the Lime Hills 1:250,000-scale quadrangles, south-central Alaska.

\section{Plate}

[Available online only at https://doi.org/10.3133/ofr20181033]

1. Legacy $\mathrm{K} / \mathrm{Ar}$ and ${ }^{40} \mathrm{Ar} / 39 \mathrm{Ar}$ Geochronologic Data from the Alaska-Aleutian Range Batholith of South-central Alaska. 


\title{
Legacy $\mathrm{K} / \mathrm{Ar}$ and ${ }^{40} \mathrm{Ar} /{ }^{39} \mathrm{Ar}$ Geochronologic Data from the Alaska-Aleutian Range Batholith of South-central Alaska
}

\author{
By Lisa L. Koeneman and Frederic H. Wilson, compilers
}

\begin{abstract}
Sample descriptions and analytical data for more than $200 \mathrm{~K} / \mathrm{Ar}$ and ${ }^{40} \mathrm{Ar} /{ }^{39} \mathrm{Ar}$ analyses from rocks of the Alaska-Aleutian Range batholith of south-central Alaska are reported here. Samples were collected over a period of 20 years by Bruce R. Reed and Marvin A. Lanphere (both U.S. Geological Survey) as part of their studies of the batholith.
\end{abstract}

\section{Introduction}

Sample descriptions and analytical data for more than $200 \mathrm{~K} / \mathrm{Ar}$ and ${ }^{40} \mathrm{Ar} /{ }^{39} \mathrm{Ar}$ analyses from rocks of the Alaska-Aleutian Range batholith on the west side of Cook Inlet in south-central Alaska are reported here (tables 1 and 2). The samples were collected by Bruce R. Reed and Marvin A. Lanphere of the U.S. Geological Survey as part of their studies of the batholith from the 1970s to early 1990s. The data included here were unpublished at the time of Bruce Reed's death in 1993; we have reconstructed sample location and rock description data from his field notes and field sheets. Analytical data are as reported in files provided by Marvin Lanphere to Bruce Reed.

Comparison of these data with existing published data and published geologic maps (Reed and Lanphere, 1969, 1972, and 1973; Wilson and others, 2015) suggests that the re-evaluation of the age assignment of a number of mapped plutons may be warranted on the basis of these newly recovered age determinations. Additionally, a pattern emerges in the overall data set (see also Wilson and others [2015] for additional data); samples in Iliamna, Kenai, and Lake Clark quadrangles tend to have a higher proportion of discordant ${ }^{1}$ age determinations (49 percent of 93 samples) relative to samples from the Lime Hills and Tyonek quadrangles (40 percent of 40 samples) (plate 1). For some samples, the discordance is extreme; some biotite samples may yield dates that are as little as half of the hornblende date. This discordance affects older and younger age determinations alike; however, it is most prevalent in samples that yield Eocene biotite ages and Late Cretaceous hornblende ages. Samples yielding Jurassic ages appear to show proportionately less discordance.

The methodology used for processing these samples is that of Faure and Mensing (2005); decay constants used are as reported by Steiger and Jager (1977).

\footnotetext{
${ }^{1}$ Discordant in this context means that within analytical error, the dates on different mineral phases do not agree. Concordant dates are generally taken to mean geologically rapid cooling and lack of disturbance to the potassium-argon system. In a few cases, where biotite yields an older date than hornblende, if the dates remain within analytical error they are still considered concordant.
} 
Table 1. Potassium-argon analytical data and descriptive information for samples of the Alaska-Aleutian Range batholith in the Tyonek, Lime Hills, Kenai, Lake Clark, and lliamna 1:250,000-scale quadrangles, south-central Alaska.

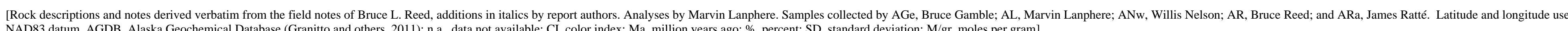

\begin{tabular}{|c|c|c|c|c|c|c|c|c|c|c|c|c|c|c|}
\hline $\begin{array}{l}\text { Map } \\
\text { no. }\end{array}$ & Sample & $\begin{array}{l}\text { Latitude } \\
\text { (degrees north) }\end{array}$ & $\begin{array}{c}\text { Longitude } \\
\text { (degrees west) }\end{array}$ & Rock type & Mineral & Method & $\% \mathrm{~K}_{2} \mathrm{O}$ & $\mathrm{SD} \__{2} \mathrm{O}$ & 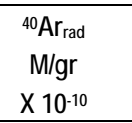 & $\%^{{ }^{*} \mathrm{~A} \text { r rad }}$ & Age (Ma) & $\begin{array}{l}\text { Error } \\
\text { (Ma) } 1 \sigma\end{array}$ & Quadrangle & Description \\
\hline 1 & 88AR 239 & 61.7356 & 154.6453 & Basalt & Whole rock & K/Ar & 0.972 & 0.001 & 0.8294 & 72.9 & 58.3 & 1.7 & Lime Hills & $\begin{array}{l}\text { Dark green and gray, fairly fresh volcanic rock; possibly andesite; possible needlles of hornblende, fresh } \\
\text { plagioclase. There is a ruund knob to the north and there may be a small volcanic center with a moat } \\
\text { between here and the topo. }\end{array}$ \\
\hline 2 & 87AR 23 & 61.4375 & 153.7042 & Granodiorite & $\begin{array}{l}\frac{\text { Biotite }}{\text { Hornblende }} \\
\end{array}$ & $\begin{array}{ll}\mathrm{K} / \mathrm{Ar} \\
\mathrm{K} / \mathrm{Ar}\end{array}$ & $\frac{7.225}{0.649}$ & 0.007 & $\frac{3.52}{0.3321}$ & $\frac{36.0}{51.7}$ & $\frac{33.5}{35.2}$ & $\frac{1.0}{1.1}$ & Lime Hills & 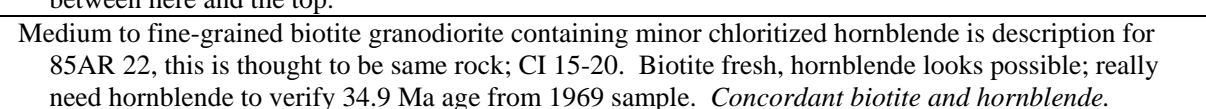 \\
\hline 3 & $87 \mathrm{AR}_{18}$ & 61.4042 & 153.6161 & Monzonite & Biotite & K/Ar & 8.910 & 0.014 & 6.517 & 77.9 & 50.1 & 1.5 & Lime Hills & 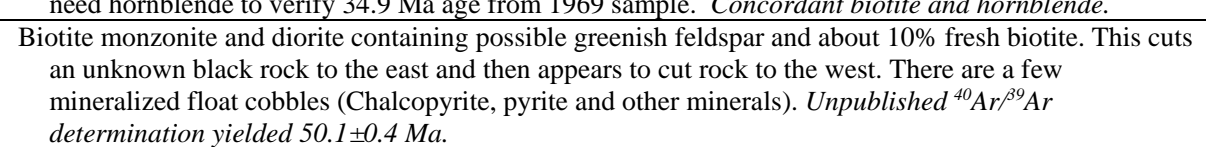 \\
\hline 4 & 81AR 141 & 61.165 & -152.6567 & Granodiorite & $\begin{array}{l}\frac{\text { Biotite }}{\text { Hornblende }} \\
\end{array}$ & $\begin{array}{l}\frac{\mathrm{K} / \mathrm{Ar}}{\mathrm{K} / \mathrm{Ar}} \\
\end{array}$ & $\frac{8.80}{0.943}$ & $\begin{array}{l}0.071 \\
0.006\end{array}$ & $\begin{array}{l}8.860 \\
1.158\end{array}$ & $\begin{array}{l}80.7 \\
82.8\end{array}$ & 68.6 & 2.1 & Tyonek & 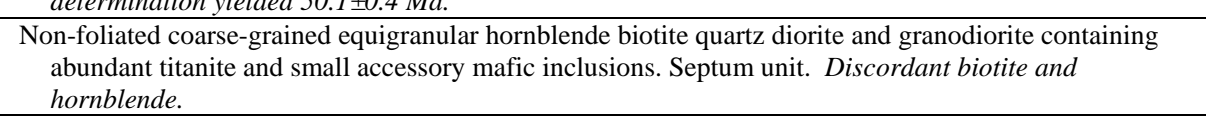 \\
\hline 5 & $81 \mathrm{AR} 91$ & 61.1427 & 152.1573 & Tonalite & $\begin{array}{l}\text { Biotite } \\
\text { Hornblende } \\
\text { Dintionde }\end{array}$ & $\begin{array}{ll}\mathrm{K} / \mathrm{Ar} \\
\mathrm{K} / \mathrm{Ar} \\
\mathrm{K} / \mathrm{s}\end{array}$ & $\begin{array}{ll}9.680 \\
0.882 \\
760\end{array}$ & $\begin{array}{ll}0.014 \\
0.006 \\
0.020\end{array}$ & $\frac{9.155}{0.8204}$ & $\frac{91.2}{72.1}$ & $\frac{64.5}{63.5}$ & $\frac{1.9}{1.9}$ & Tyonek & $\begin{array}{l}\text { Biotitithornthblende quartz diorite containing itanite. Summit Lake sequence. Concordant biotite and } \\
\text { horbblende, where biotite is slightly older. }\end{array}$ \\
\hline$\frac{6}{7}$ & $\begin{array}{l}87 \mathrm{AR} 76 \\
85 \mathrm{AR} 21\end{array}$ & $\begin{array}{l}61.1356 \\
61.1194\end{array}$ & $\begin{array}{l}153.7164 \\
152.9\end{array}$ & $\begin{array}{l}\text { Granodionite } \\
\text { Quartz monzodiorite }\end{array}$ & $\begin{array}{l}\text { Biotite } \\
\text { Biotite }\end{array}$ & $\begin{array}{l}\mathrm{K} / \mathrm{Ar} \\
\mathrm{K} / \mathrm{Ar}\end{array}$ & $\frac{7.660}{8.685}$ & 0.028 & $\begin{array}{l}4.466 \\
7.324\end{array}$ & $\begin{array}{l}61.0 \\
72.9\end{array}$ & $\frac{40.0}{57.6}$ & $\frac{1.2}{1.7}$ & $\begin{array}{l}\frac{\text { Lime Hills }}{\text { Tyonek }} \\
\end{array}$ & 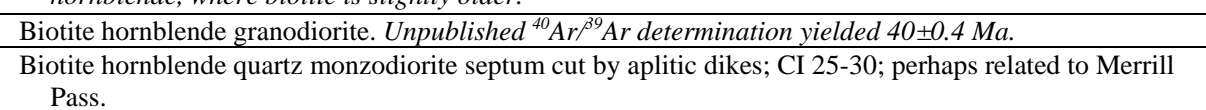 \\
\hline 8 & $85 \mathrm{AR} 22$ & 61.0972 & 152.9417 & Tonalite & $\begin{array}{l}\text { Biotite } \\
\text { Hornblende }\end{array}$ & $\begin{array}{l}\mathrm{K} / \mathrm{Ar} \\
\mathrm{K} / \mathrm{Ar}\end{array}$ & $\begin{array}{l}7.350 \\
0.586\end{array}$ & $\begin{array}{l}0.014 \\
0.001\end{array}$ & $\frac{6.213}{0.5279}$ & $\begin{array}{l}79.1 \\
33.4\end{array}$ & $\begin{array}{ll}57.8 \\
61.6\end{array}$ & $\frac{1.7}{1.8}$ & Tyonek & 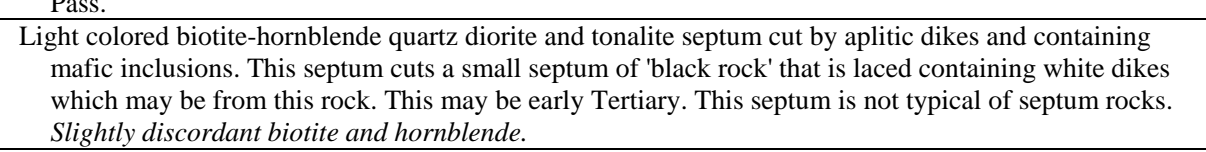 \\
\hline$\frac{9}{10}$ & $\begin{array}{l}85 \mathrm{AR} 42 \\
85 \mathrm{AR} 47\end{array}$ & $\begin{array}{l}61.0944 \\
61.0944\end{array}$ & $\frac{153.5244}{153.5244}$ & $\begin{array}{l}\text { Granite } \\
\text { Granodiorite }\end{array}$ & \begin{tabular}{|l} 
Biotite \\
Biotite
\end{tabular} & $\begin{array}{l}\mathrm{K} / \mathrm{Ar} \\
\mathrm{K} / \mathrm{Ar}\end{array}$ & $\begin{array}{l}9.16 \\
8.745\end{array}$ & 0.042 & 5.045 & $\begin{array}{l}70.9 \\
71.3\end{array}$ & $\begin{array}{l}37.9 \\
44.6\end{array}$ & $\frac{1.1}{1.3}$ & $\begin{array}{l}\text { Lime Hills } \\
\text { Lime Hills }\end{array}$ & 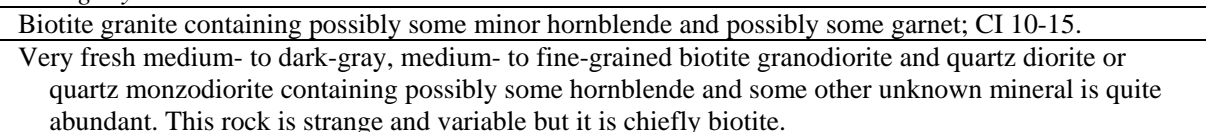 \\
\hline 11 & 85AR 23 & 61.0918 & 152.9889 & Quartz monzodiorite & Hornblende & K/Ar & 0.631 & 0.009 & 0.6038 & 53.4 & 65.3 & 2.0 & Tyonek & 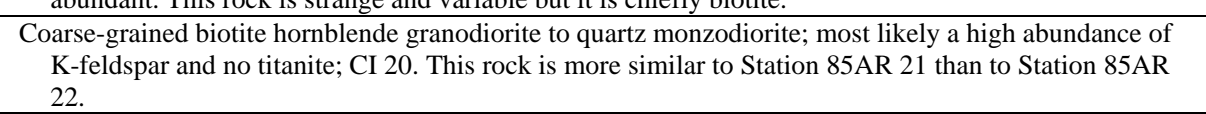 \\
\hline 12 & $81 \mathrm{AR} 53$ & 61.05 & 152.1583 & Tonalite & $\begin{array}{l}\frac{\text { Biotite }}{\text { Hornblende }} \\
\end{array}$ & $\begin{array}{ll}\mathrm{K} / \mathrm{Ar} \\
\mathrm{K} / \mathrm{Ar}\end{array}$ & $\begin{array}{l}8.895 \\
1.007\end{array}$ & 0.006 & $\begin{array}{l}9.29 \\
1.034 \\
\end{array}$ & $\begin{array}{l}83.0 \\
51.7\end{array}$ & $\frac{71.1}{69.9}$ & $\frac{2.1}{2.1}$ & Tyonek & $\begin{array}{l}\text { 2.2. } \\
\text { Hornblende-biotite quartz diorite containing titanite; Cl is about 30-35; appears to be Cretaceous. } \\
\text { Blockade sequence. Discordant bioitite and hornblende where biotite is older. }\end{array}$ \\
\hline$\frac{13}{14}$ & $\begin{array}{l}85 \mathrm{AR} 39 \\
85 \mathrm{AR} 25\end{array}$ & $\begin{array}{l}61.0383 \\
61.0167\end{array}$ & $\begin{array}{l}153.7317 \\
153.0025\end{array}$ & $\begin{array}{l}\text { Granodiorite } \\
\text { Granodiorite }\end{array}$ & $\begin{array}{l}\text { Hornblende } \\
\text { Biotite }\end{array}$ & $\begin{array}{l}\mathrm{K} / \mathrm{Ar} \\
\mathrm{K} / \mathrm{Ar}\end{array}$ & $\begin{array}{l}0.756 \\
8.995\end{array}$ & 0.001 & 0.883 & $\begin{array}{l}41.8 \\
73.4\end{array}$ & $\begin{array}{l}79.4 \\
57.5\end{array}$ & $\begin{array}{l}1.4 \\
1.7\end{array}$ & $\begin{array}{l}\text { Lime Hills } \\
\text { Lime Hills }\end{array}$ & 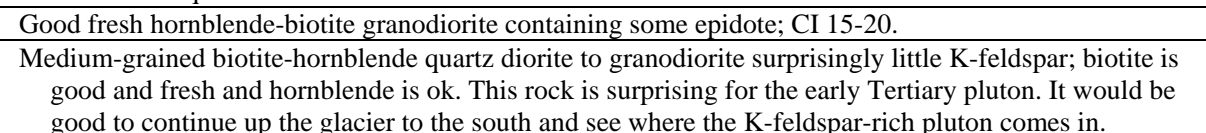 \\
\hline 15 & 78AR 218 & 60.996 & 153.0687 & Granodiorite & $\begin{array}{l}\text { Biotite } \\
\text { Horblende }\end{array}$ & $\begin{array}{l}\mathrm{K} / \mathrm{Ar} \\
\mathrm{K} / \mathrm{Ar}\end{array}$ & 9.255 & 0.007 & $\begin{array}{l}7.724 \\
0.4733\end{array}$ & $\frac{86.3}{45.4}$ & $\frac{57.1}{56.5}$ & $\frac{1.1}{1.7}$ & Lake Clark & 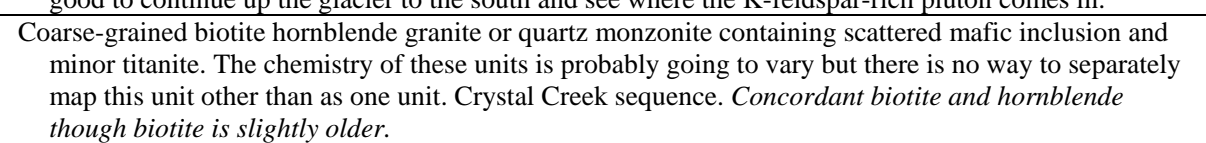 \\
\hline 16 & 77AR 170 & 60.9914 & 153.3251 & Granodiorite & Hornblende & $\begin{array}{c}\text { K/Ar } \\
\text { weighted } \\
\text { mean }\end{array}$ & 0.463 & 0.013 & $\begin{array}{l}0.2185 \\
0.2290\end{array}$ & $\begin{array}{l}35.2 \\
26.1\end{array}$ & $\begin{array}{l}32.5 \\
34.0\end{array}$ & $\begin{array}{l}1.0 \\
1.0 \\
0.7\end{array}$ & Lake Clark & $\begin{array}{l}\text { Biotite and hornblendende quartzr dioirite/granodionite containing mafic inclusion; same rock as } 77 \mathrm{AR} 169 . \\
\text { Neacola unit. Weighted mean of two determinations. }\end{array}$ \\
\hline 17 & 81AR 101 & 60.9793 & 152.1187 & Tonalite & $\begin{array}{l}\text { Biotite } \\
\text { Hornblende }\end{array}$ & $\begin{array}{l}\text { K/Ar } \\
\text { K/Ar }\end{array}$ & 9.610 & $\begin{array}{ll}0.014 \\
0.002\end{array}$ & $\begin{array}{ll}10.91 \\
0.8706\end{array}$ & $\begin{array}{l}90.1 \\
88.4\end{array}$ & 77.2 & $\frac{2.3}{2.4}$ & Kenai & $\begin{array}{l}\text { Slightly foliated biotite hornblende granodiorite containing titanite; CI 25. Blockade sequence. } \\
\text { Concordant biotite and horbllende. }\end{array}$ \\
\hline 18 & 77AR 165 & 60.9727 & 153.691 & Granodiorite & $\begin{array}{l}\text { Biotite } \\
\text { Hornblende }\end{array}$ & $\begin{array}{c}\text { K/Ar } \\
\text { Weighted } \\
\text { mean }\end{array}$ & $\frac{9.125}{0.732}$ & $\begin{array}{ll}0.021 \\
0.005\end{array}$ & $\begin{array}{l}4.1100 \\
0.2886 \\
0.3303\end{array}$ & $\begin{array}{l}41.8 \\
47.0 \\
47.8\end{array}$ & $\begin{array}{l}31 \\
27.1 \\
31.0 \\
28.8\end{array}$ & $\begin{array}{l}0.9 \\
0.8 \\
0.9 \\
0.6\end{array}$ & Lake Clark & $\begin{array}{l}\text { Very fresh hornblende bioite quartz diorite or granodiorite; CI 21.5-22.55 same rock as } 77 \mathrm{AR} 162 \text { and } \\
77 \mathrm{RR} 163 \text {; belt along west flank of mountains. Discordant biotite and hornblende where biotite is } \\
\text { older. }\end{array}$ \\
\hline 19 & 77AR 166 & 60.9673 & 153.5793 & Granodiorite & Biotite & $\begin{array}{c}\text { K/Ar } \\
\begin{array}{c}\text { weighted } \\
\text { mean }\end{array} \\
\end{array}$ & 9.310 & 0.043 & $\begin{array}{l}4.279 \\
4.306\end{array}$ & $\begin{array}{l}79.8 \\
66.2\end{array}$ & $\begin{array}{l}31.6 \\
31.8\end{array}$ & $\begin{array}{l}0.0 \\
0.9 \\
1.0 \\
0.7\end{array}$ & Lake Clark & $\begin{array}{l}\text { Biotite hornblende quartz diorite/granodioritit; good fresh rock forms massive cliffs to the north } \\
\text { containing a few mafic inclusions; CI 12-15. Neacola unit. Weighted mean of two determinations. }\end{array}$ \\
\hline 20 & 81AR 126 & 60.94 & 152.1288 & Granodiorite & Biotite & $\mathrm{K} / \mathrm{Ar}$ & 9.075 & 0.007 & 10.89 & 90.7 & 81.5 & 2.4 & Kenai & $\begin{array}{l}\text { Foliated biotite granodiorite and trondhjemite possibly containing some garnet. The rocks all along the } \\
\text { low hills are the same. } A G D B \text { reports trondhjemite. Assigned to trondhjimite unit. }\end{array}$ \\
\hline 21 & $81 \mathrm{AR} 96$ & 60.9381 & 152.3521 & Tonalite & $\begin{array}{l}\frac{\text { Biotite }}{\text { Hornblende }} \\
\end{array}$ & $\begin{array}{ll}\mathrm{K} / \mathrm{Ar} \\
\mathrm{K} / \mathrm{Ar} \\
\end{array}$ & $\frac{9.340}{1.066}$ & 0.014 & $\begin{array}{l}9.717 \\
1.109 \\
\end{array}$ & $\frac{86.8}{67.4}$ & $\frac{70.9}{70.9}$ & $\frac{2.1}{2.1}$ & Kenai & 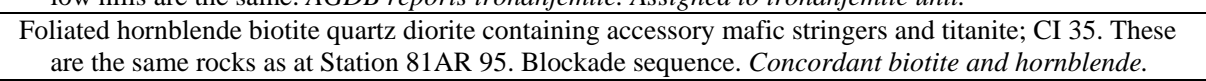 \\
\hline 22 & 78AR 227 & 60.9043 & 153.7623 & Quartz diorite & $\begin{array}{ll}\text { Bioitite } \\
\text { Homblende. }\end{array}$ & $\begin{array}{lll}\text { K/Ar } \\
\text { K/ar }\end{array}$ & 8.32 & 0.028 & $\frac{7.74}{0.617}$ & $\frac{79.7}{7936}$ & $\frac{63.5}{615}$ & $\frac{1.9}{1.9}$ & Lake Clark & 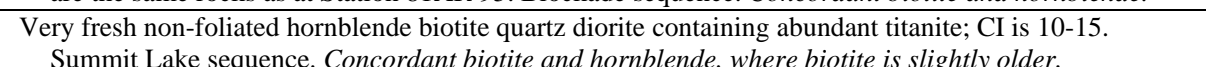 \\
\hline 23 & 78AR 224 & 60.8989 & 152.9481 & Quartz diorite & Biotite & $\mathrm{K} / \mathrm{Ar}$ & 8.745 & 0.008 & 8.586 & 77.0 & 66.9 & 2.0 & Kenai & 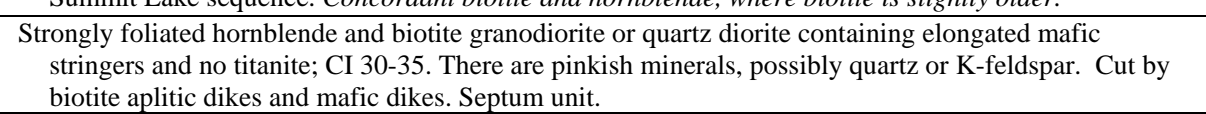 \\
\hline 24 & 81AR 135 & 60.8882 & 152.3324 & Trondhjemite & $\begin{array}{c}\frac{\text { Biotite }}{\text { Hormblende }} \\
\end{array}$ & $\begin{array}{ll}\mathrm{K} / \mathrm{Ar} \\
\mathrm{K} / \mathrm{Ar}\end{array}$ & $\frac{9.00}{0.612}$ & $\frac{0.057}{0.008}$ & $\frac{9.919}{1.223}$ & $\frac{73.7}{73.6}$ & $\frac{75.00}{134.0}$ & $\frac{2.25}{4.0}$ & Kenai & 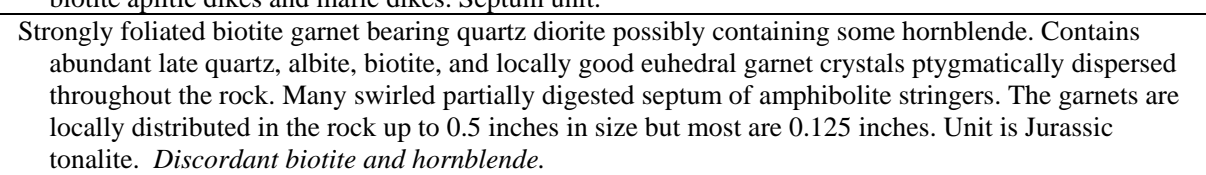 \\
\hline 25 & 78AR 304 & 60.8865 & 153.5689 & Aplite & Biotite & K/Ar & 8.945 & 0.008 & 2.685 & 69.7 & 20.7 & 0.6 & Lake Clark & 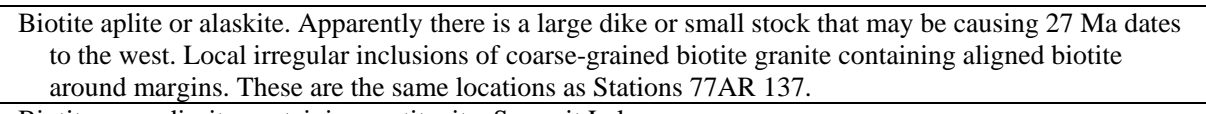 \\
\hline 26 & $\begin{array}{ll}\text { 81AR } 45 \\
77 \mathrm{AR} 134\end{array}$ & $\begin{array}{ll}60.8829 \\
60.8714\end{array}$ & $\begin{array}{ll}152.8133 \\
153.637\end{array}$ & $\begin{array}{cc}\text { Granodiorite } \\
\text { Granodiorite }\end{array}$ & $\begin{array}{l}\text { Biotite } \\
\text { Biotite }\end{array}$ & $\begin{array}{ll}\mathrm{K} / \mathrm{Ar} \\
\mathrm{K} / \mathrm{Ar} \\
\end{array}$ & $\begin{array}{l}9.275 \\
8.970\end{array}$ & $\begin{array}{ll}0.008 \\
0.015\end{array}$ & $\frac{9.754}{2.808}$ & $\frac{84.3}{59.0}$ & $\begin{array}{ll}71.6 \\
21.6\end{array}$ & 2.1 & $\begin{array}{l}\text { Kenai } \\
\text { Lake Clark }\end{array}$ & $\begin{array}{l}\text { Biotite granodioritit containing no titanite.e Summit Lake sequence. } \\
\text { Very fresh hornblende granodiorite; CI 18-20; probably the same as } 77 \text { AR } 133 \text { but does not look like }\end{array}$ \\
\hline & & & & & Hornblende & K/Ar & 0.466 & 0.019 & 0.1916 & 23.2 & 28.3 & 0.8 & & $\begin{array}{l}\text { Merrill Pass, though assigned to Merrill Pass sequence. Discordant biotite and hornblende. } \\
\text { a }\end{array}$ \\
\hline
\end{tabular}




\begin{tabular}{|c|c|c|c|c|c|c|c|c|c|c|c|c|c|c|}
\hline $\begin{array}{l}\text { Map } \\
\text { no. }\end{array}$ & Sample & $\begin{array}{c}\text { Latitude } \\
\text { (degrees north) }\end{array}$ & $\begin{array}{c}\text { Longitude } \\
\text { (degrees west) }\end{array}$ & Rock type & Mineral & Method & $\% \mathrm{~K}_{2} \mathrm{O}$ & SD_K $\mathrm{K}_{2} \mathrm{O}$ & $\begin{array}{l}40 \mathrm{Ar} \text { rad } \\
\text { MIgr } \\
\times 10^{-10} \\
\end{array}$ & $\%^{0} \mathrm{AA}$ rad & Age (Ma) & $\begin{array}{c}\text { Error } \\
\text { (Ma) } 1 \sigma\end{array}$ & Quadrangle & Description \\
\hline 28 & 81AR 137 & 60.8678 & 153.0834 & Granodiorite & $\frac{\text { Biotite }}{\text { Hormblende }}$ & 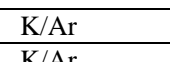 & 8.165 & $\frac{0.035}{0.004}$ & 9.568 & 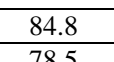 & $\frac{79.6}{994}$ & $\frac{2.4}{2.7}$ & Lake Clark & $\begin{array}{l}\text { Biotitite hormblende granodiorite containing abundant titanite and rounded mafic inclusions. This is good } \\
\text { reck }\end{array}$ \\
\hline 29 & 81AR 116 & 60.8677 & 152.3316 & Tonalite & $\begin{array}{l}\text { Biotite } \\
\text { Hormblende }\end{array}$ & $\frac{\mathrm{K} / \mathrm{Ar}}{\mathrm{K} / \mathrm{Ar}}$ & $\frac{9.200}{1.146}$ & 0.014 & $\frac{0.1791}{23.58}$ & $\frac{18.5}{93.5}$ & $\begin{aligned} 91.4 \\
99.7 \\
155.8\end{aligned}$ & $\begin{array}{ll}2.7 \\
3.0 \\
4.7\end{array}$ & Kenai & 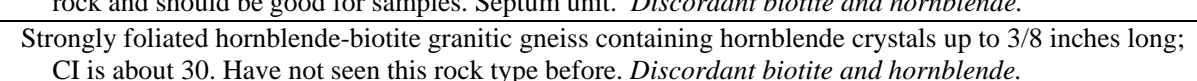 \\
\hline 30 & 77AR 140 & 60.8546 & 153.601 & Quartz monzonite & Biotite & $\begin{array}{c}\text { K/Ar } \\
\substack{\text { weighted } \\
\text { mean }}\end{array}$ & 8.210 & 0.015 & $\begin{array}{ll}2.65 \\
2.60\end{array}$ & $\begin{array}{l}79.6 \\
56.8\end{array}$ & $\begin{array}{l}22.3 \\
21.9 \\
22.1\end{array}$ & $\begin{array}{l}0.7 \\
0.7 \\
0.5\end{array}$ & Lake Clark & Biotite hornblende quartz monzonite; Merrill Pass sequence. Weighted mean of two determinations. \\
\hline 31 & 81AR 152 & 60.833 & 152.2643 & Tonalite & Hormblende & $\begin{array}{l}\mathrm{K} / \mathrm{Ar} \\
\begin{array}{c}\text { weighted } \\
\text { mean }\end{array} \\
\mathrm{K} / \mathrm{Ar}\end{array}$ & 8.84 & 0.015 & $\begin{array}{l}20.08 \\
20.31\end{array}$ & $\begin{array}{l}87.9 \\
95.5\end{array}$ & $\begin{array}{l}151 \\
153 \\
152.0 \\
151\end{array}$ & $\begin{array}{l}4.5 \\
4.6 \\
3.2 \\
4.65\end{array}$ & Kenai & $\begin{array}{l}\text { Good fresh hornblende granodiorite; CI 25; appears to be Jurassic. Concordant biotite and hornblende. } \\
\text { Weighted mean of two biotite determinations. }\end{array}$ \\
\hline 32 & $\begin{array}{l}78 \mathrm{ANw} 205 \\
78 \mathrm{AR} 291\end{array}$ & $\begin{array}{l}60.8253 \\
60.7632\end{array}$ & $\begin{array}{l}154.4965 \\
152.5132\end{array}$ & $\begin{array}{l}\text { Granite } \\
\text { Quartz diorite }\end{array}$ & $\begin{array}{l}\text { Biotite } \\
\text { Biotite }\end{array}$ & $\begin{array}{l}\text { K/Ar } \\
\text { K/Ar }\end{array}$ & $\begin{array}{l}8.960 \\
9.045\end{array}$ & $\begin{array}{ll}0.028 \\
0.007\end{array}$ & $\begin{array}{c}8.241 \\
18.54\end{array}$ & $\begin{array}{l}60.7 \\
93.1\end{array}$ & $\begin{array}{c}62.8 \\
137\end{array}$ & $\begin{array}{ll}1.9 \\
4.1\end{array}$ & $\begin{array}{l}\text { Lake Clark } \\
\text { Kenai }\end{array}$ & $\begin{array}{l}\text { Biotite granite. } \\
\text { Slightly folited leucocoratic biotite quartz diorite or possibly granodionite containing some epidote; CI is } \\
\text { about 8-10. The biotite locally is gone to a pale green chlorite. Assigned to trondhiemite unit. }\end{array}$ \\
\hline 34 & 77AR 141 & 60.7516 & 153.7975 & Granodiorite & Hornblende & $\mathrm{K} / \mathrm{Ar}$ & 1.128 & 0.026 & 0.8208 & 70.6 & 49.8 & 1.5 & Lake Clark & $\begin{array}{l}\text { Turquoise stock. Horblende granite; defininitly cuts Tertiary sub-horizontal basalts. CI 8-10. Peralkaline } \\
\text { granite unit. }\end{array}$ \\
\hline 35 & $81 \mathrm{AR} 20$ & 60.7317 & 152.3787 & Tonalite & Hornblende & $\begin{array}{c}\text { K/Ar } \\
\text { weighted } \\
\text { mean } \\
\text { K/Ar } \\
\text { weighted } \\
\text { mean }\end{array}$ & 0.602 & 0.008 & $\begin{array}{l}1.381 \\
1.316\end{array}$ & $\begin{array}{l}83.5 \\
44.1\end{array}$ & $\begin{array}{l}169 \\
170 \\
169.5 \\
153 \\
146 \\
149.3\end{array}$ & $\begin{array}{l}5.1 \\
5.1 \\
3.6 \\
4.6 \\
4.4\end{array}$ & Kenai & $\begin{array}{l}\text { Nice fresh hornblende biotite quartz diorite containing no titanite; } 50 / 50 \text { hornblendel/iotite; CI is about } \\
\text { 15. All the rocks around the small pond appear to be the same. Uniti is Jurassic tonalite. Weighted } \\
\text { mean of fwo biotite and hornblende determinations. Discordant biotite and hornblende where biotite is } \\
\text { older. }\end{array}$ \\
\hline 36 & 78AR 223 & 60.7299 & 153.18 & Quartz diorite & $\frac{\text { Biotite }}{\text { Hormblende }}$ & $\frac{\mathrm{K} / \mathrm{Ar}}{\mathrm{K} / \mathrm{Ar}}$ & $\frac{7.375}{0.697}$ & $\frac{0.021}{0.002}$ & $\begin{array}{l}8.132 \\
0.8885\end{array}$ & $\frac{84.3}{81.75}$ & $\frac{756}{756}$ & $\frac{2.2}{2.6}$ & Lake Clark & 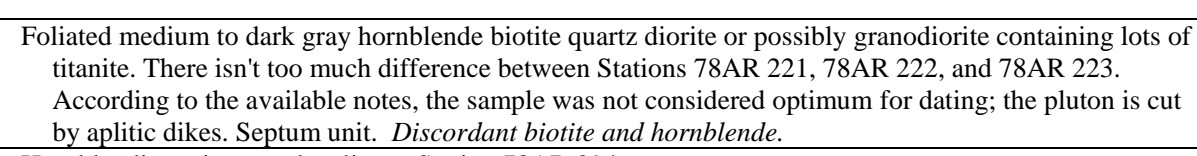 \\
\hline$\frac{37}{38}$ & $\begin{array}{l}81 \mathrm{AR} 44 \\
77 \mathrm{AR} 117\end{array}$ & $\begin{array}{ll}60.718 \\
60.6778\end{array}$ & $\begin{array}{l}152.5922 \\
153.4145\end{array}$ & $\begin{array}{l}\text { Hornblendite } \\
\text { Quartz diorite }\end{array}$ & $\begin{array}{c}\frac{\text { Hornblende }}{3 \text { Biotite }} \\
\text { Hormblende }\end{array}$ & $\begin{array}{l}\mathrm{K} / \mathrm{Ar} \\
\mathrm{K} / \mathrm{Ar} \\
\mathrm{K} / \mathrm{Ar}\end{array}$ & $\begin{array}{l}0.357 \\
9.205 \\
0.876\end{array}$ & $\begin{array}{ll}0.001 \\
0.035 \\
0.001\end{array}$ & $\begin{array}{ll}0.7785 \\
11.80 \\
1.079\end{array}$ & $\begin{array}{l}53.6 \\
86.0 \\
77.2\end{array}$ & $\begin{array}{ll}\frac{146}{86.9} \\
83.6\end{array}$ & $\begin{array}{ll}\frac{4.4}{2.6} \\
2.5\end{array}$ & $\begin{array}{ll}\text { Kenai } \\
\text { Lake Clark }\end{array}$ & 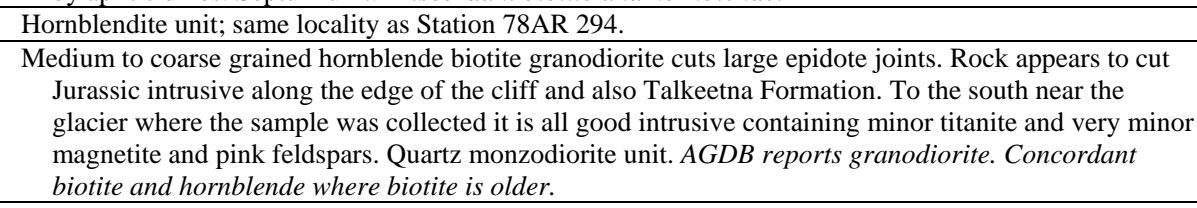 \\
\hline 39 & $78 \mathrm{AR} 268$ & 60.6605 & 153.3307 & Granodiorite & $\frac{\text { Biotite }}{\text { Hormblende }}$ & $\begin{array}{l}\text { K/Ar } \\
\text { KAr }\end{array}$ & 8.315 & $\frac{0.049}{0005}$ & 10.53 & $\begin{array}{c}74.5 \\
791\end{array}$ & 85.9 & $\frac{2.6}{2.5}$ & Lake Clark & 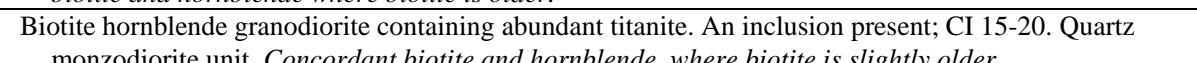 \\
\hline 40 & 78AR 282 & 60.657 & 152.7258 & Trondhjemite & Muscovite & $\mathrm{K} / \mathrm{Ar}$ & 10.18 & 0.0 & 21.69 & 93.7 & 142 & 4.3 & Kenai & 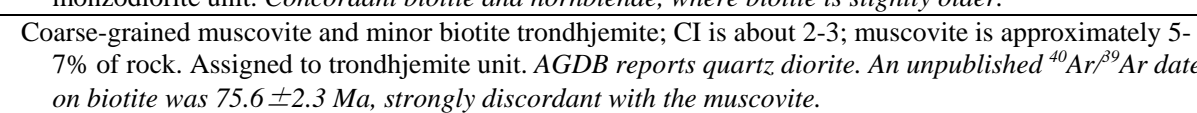 \\
\hline 41 & 77AR 115 & 60.6415 & 153.4369 & Quartz diorite & Hornblende & K/Ar & 0.369 & 0.002 & 0.1802 & 42.3 & 33.5 & 1.0 & Lake Clark & 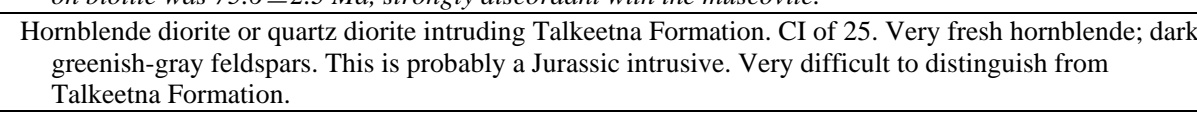 \\
\hline 42 & 78AR 283 & 60.6354 & 152.6683 & $\begin{array}{l}\text { Granodiorite } \\
\end{array}$ & $\begin{array}{c}\text { Biotite } \\
\text { Hornblende }\end{array}$ & $\begin{array}{ll}\mathrm{K} / \mathrm{Ar} \\
\mathrm{K} / \mathrm{Ar}\end{array}$ & $\begin{array}{ll}9.205 \\
0.692\end{array}$ & $\begin{array}{lll}0.007 \\
0.014\end{array}$ & $\begin{array}{ll}19.68 \\
1.417\end{array}$ & $\begin{array}{ll}94.6 \\
88.2\end{array}$ & $\frac{142}{137}$ & $\begin{array}{ll}4.3 \\
4.1\end{array}$ & Kenai & $\begin{array}{l}\text { Medium-grained medium-gray hornblende biotite quartz diorite containing a medium amount of titanite; } \\
\text { CI I s about 20. Uniti is Jurassist tonalitit. These rocks look like the Summitit Lake rocks from north of } \\
\text { Lake Clark Pass. AGDB reports quartz diorite. Discordant biotite and hornblende where biotite is } \\
\text { older. }\end{array}$ \\
\hline 43 & 77AR 192 & 60.6243 & 152.9395 & Granodiorite & $\begin{array}{c}\text { Biotite } \\
\text { Hornblende }\end{array}$ & $\begin{array}{ll}\mathrm{K} / \mathrm{Ar} \\
\mathrm{K} / \mathrm{Ar}\end{array}$ & $\frac{8.935}{0.771}$ & $\frac{0.021}{0.004}$ & $\begin{array}{ll}9.041 \\
0.7967\end{array}$ & $\begin{array}{ll}76.1 \\
51.9\end{array}$ & $\frac{69.3}{70.4}$ & $\frac{2.1}{2.1}$ & Kenai & 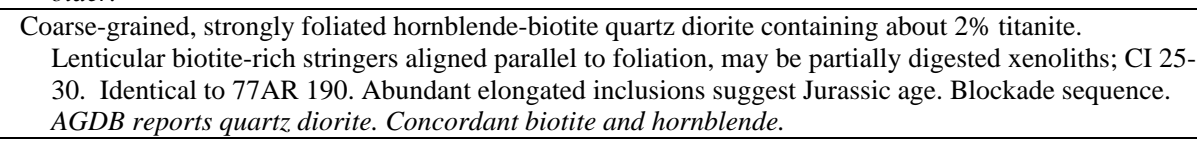 \\
\hline 44 & 77AR 129 & 60.6199 & 153.5299 & Quartz monzonite & Biotite & K/Ar & 9.045 & 0.007 & 5.272 & 43.2 & 40 & 1.2 & Lake Clark & 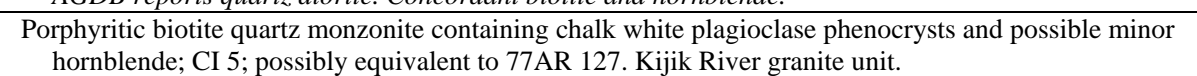 \\
\hline 45 & 72AR 146 & 60.6018 & 152.8996 & Quartz diorite & $\frac{\text { Biotite }}{\text { Hormblende }}$ & $\begin{array}{l}\text { K/Ar } \\
\text { KAr }\end{array}$ & $\begin{array}{l}8.86 \\
0609\end{array}$ & 0.014 & \begin{tabular}{|l|l|}
10.14 \\
07171
\end{tabular} & $\begin{array}{l}54.2 \\
472 \\
\end{array}$ & 77.8 & $\frac{2.3}{2.4}$ & Kenai & 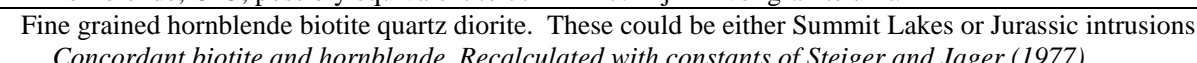 \\
\hline 46 & 78AR 327 & 60.5919 & 153.1682 & Quartz diorite & $\frac{\text { Bintite }}{\text { Hornblende }}$ & $\begin{array}{ll}\frac{\mathrm{N} / \mathrm{Ar}}{\mathrm{K}} \\
\mathrm{K} / \mathrm{Ar}\end{array}$ & $\begin{array}{l}7.175 \\
0.392 \\
\end{array}$ & $\begin{array}{ll}0.007 \\
0.002\end{array}$ & $\begin{array}{ll}6.263 \\
0.363 \\
\end{array}$ & $\begin{array}{l}3.3 \\
56.7 \\
56.7\end{array}$ & $\begin{aligned} 59.6 \\
63.1 \\
63\end{aligned}$ & $\begin{array}{l}1.8 \\
1.9 \\
\end{array}$ & Lake Clark & Blockade sequence. Biotite $\mathrm{K}_{2} \mathrm{O}$ only $7.33 \%$. Concordant biotite and hornblende. \\
\hline 47 & 78AR 172 & 60.591 & 153.6049 & Quartz monzonite & 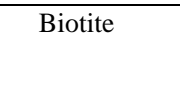 & K/Ar & 8.415 & 0.021 & 4.914 & 63.8 & 40.1 & 1.2 & Lake Clark & 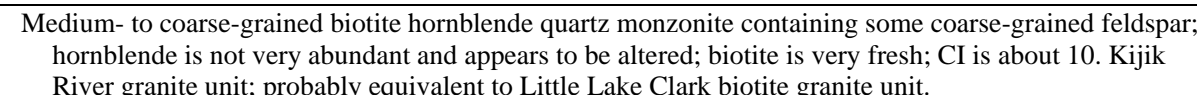 \\
\hline 48 & $81 \mathrm{AR} 7$ & 60.5573 & 152.4562 & Quartz monzonite & Biotite & K/Ar & 6.865 & 0.036 & 18.02 & 94.6 & 174 & 5.2 & Kenai & $\begin{array}{l}\text { Good coarse-grained biotite quartz monzonite containing decent minor hormblende; looks to be mafic } \\
\text { intrusuive. II i f possibly part of the Talkeetna Formation. Assigned to trondhjemite unit. Biotite } K_{2} O \\
\text { only } 6.865 \% \text {. }\end{array}$ \\
\hline 49 & 78AR 105 & 60.5476 & 153.4212 & Quartz diorite & $\frac{\text { Biotite }}{\text { Hormblende }}$ & $\begin{array}{ll}\mathrm{K} / \mathrm{Ar} \\
\mathrm{K} / \mathrm{Ar}\end{array}$ & $\frac{8.045}{0.918}$ & $\frac{0.008}{0.006}$ & $\begin{array}{ll}6.425 \\
1.089\end{array}$ & $\begin{array}{ll}74.9 \\
72.8\end{array}$ & $\frac{54.6}{80.6}$ & $\frac{1.6}{2.4}$ & Lake Clark & 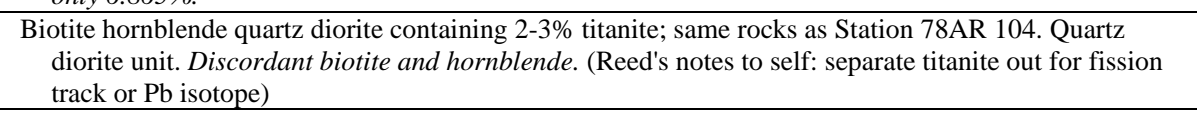 \\
\hline 50 & 78AR 111 & 60.5316 & 153.3268 & Alaskite & $\begin{array}{c}\text { Biotite } \\
\text { Hornblende }\end{array}$ & $\begin{array}{cl}\mathrm{K} / \mathrm{Ar} \\
\mathrm{K} / \mathrm{Ar}\end{array}$ & $\begin{array}{l}9.495 \\
1.211\end{array}$ & $\begin{array}{lll}0.021 \\
0.011\end{array}$ & \begin{tabular}{|l|}
9.096 \\
1.240
\end{tabular} & $\begin{array}{l}74.0 \\
68.6\end{array}$ & $\begin{array}{l}665.3 \\
69.7\end{array}$ & $\begin{array}{l}2.0 \\
2.1\end{array}$ & Lake Clark & $\begin{array}{l}\text { Extremely coarse grained biotite granite containing minor horblende. It looks similar to the Lake Clark } \\
\text { graninte but has sornblende and K-feldspar crystals up to } 1.5 \text { inches long. Island unit. Discordant } \\
\text { biotite and hornblende. }\end{array}$ \\
\hline 51 & 81AR 182 & 60.5267 & $\begin{array}{l}152.4567 \\
\end{array}$ & Quartz monzonite & Biotite & K/Ar & 7.905 & 0.007 & 20.51 & 87.8 & 172 & 5.2 & Kenai & $\begin{array}{l}\text { Quartz monzonite body south of the Drift River. Biotite quartz monzonite containing little to no } \\
\text { hornnlende biotite is the only dateable mineral; } \mathrm{CI} \text { is about } 1 .\end{array}$ \\
\hline 52 & 77AR 203 & 60.5246 & 153.4862 & Quartz diorite & $\begin{array}{l}\text { Biotite } \\
\text { Hornblende }\end{array}$ & $\begin{array}{l}\mathrm{K} / \mathrm{Ar} \\
\mathrm{K} / \mathrm{Ar}\end{array}$ & $\begin{array}{l}8.910 \\
0.812\end{array}$ & $\begin{array}{l}0.042 \\
0.001\end{array}$ & \begin{tabular}{|l|}
4.861 \\
0.9308
\end{tabular} & $\begin{array}{l}77.1 \\
79.3\end{array}$ & $\begin{array}{l}37.5 \\
77.9\end{array}$ & $\frac{1.1}{2.3}$ & Lake Clark & 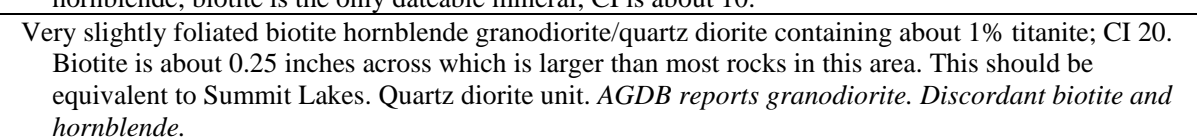 \\
\hline 53 & 72AR 293 & 60.5162 & 153.33 & Quartz monzonite & Biotite & K/Ar & 9.140 & 0.015 & 8.92 & 90.5 & 66.6 & 2.0 & Lake Clark & $\begin{array}{l}\text { Biotite quartz monzonite containing minor hornblende. This may be Merrill Pass sequence. It could also } \\
\text { be equivalent to } 72 A R \text { 292. AGDB calls it granodiorite. Recalculated with constants of Steiger and } \\
\text { Jager (1977). }\end{array}$ \\
\hline
\end{tabular}




\begin{tabular}{|c|c|c|c|c|c|c|c|c|c|c|c|c|c|c|}
\hline $\begin{array}{l}\text { Map } \\
\text { no. }\end{array}$ & Sample & $\begin{array}{c}\text { Latitude } \\
\text { (degrees north) }\end{array}$ & $\begin{array}{l}\text { Longitude } \\
\text { (degrees west) }\end{array}$ & Rock type & Mineral & Method & $\% \mathrm{k}_{2} 0$ & SD $\mathrm{K}_{2} \mathrm{O}$ & $\begin{array}{l}\text { AAArad } \\
\text { MMgr } \\
\times 10010\end{array}$ & 9\%"A Araa & Age (Ma) & $\begin{array}{l}\text { Error } \\
\text { (Ma) } 1 \sigma\end{array}$ & Quadrangle & Description \\
\hline 54 & 78AR 152 & 60.5146 & 152.9544 & $\begin{array}{l}\text { Granodiorite } \\
\mathrm{C}\end{array}$ & $\begin{array}{l}\text { Muscovite } \\
\text { Biotite }\end{array}$ & $\begin{array}{c}\text { K/Ar } \\
\text { K/Ar } \\
\begin{array}{c}\text { weighted } \\
\text { mean }\end{array}\end{array}$ & $\frac{10.325}{9.105}$ & $\begin{array}{l}0.049 \\
0.008\end{array}$ & $\begin{array}{l}21.78 \\
15.48 \\
15.43\end{array}$ & $\begin{array}{l}96.5 \\
79.4 \\
84.1\end{array}$ & $\begin{array}{l}141 \\
114 \\
114 \\
114\end{array}$ & $\begin{array}{l}4.2 \\
3.4 \\
3.4 \\
2.4\end{array}$ & Kenai & $\begin{array}{l}\text { Leucococratic biotite granodiorite containing small pegmatite veins have garnet and muscovite; CI is } \\
\text { relatively } 5 \text { 5-8. Addacent to Redoubt Volcano. Assigned to trondhjemite unit. Weighted mean of two } \\
\text { biotite determinations. Discordant biotite and muscovite. }\end{array}$ \\
\hline 55 & $81 \mathrm{AR} 30$ & 60.5127 & 152.5801 & Tonalite & 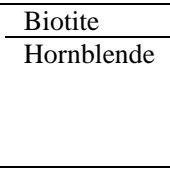 & $\begin{array}{l}\text { K/Ar } \\
\text { K/Ar } \\
\text { weighted } \\
\text { mean }\end{array}$ & $\begin{array}{l}8.855 \\
0.669\end{array}$ & $\begin{array}{l}0.009 \\
0.007\end{array}$ & $\begin{array}{l}22.91 \\
1.61 \\
1.60\end{array}$ & $\begin{array}{l}89.1 \\
78.9 \\
68.1\end{array}$ & $\begin{array}{l}171 \\
160 \\
159 \\
159.5\end{array}$ & $\begin{array}{l}5.1 \\
4.8 \\
4.8 \\
3.4\end{array}$ & Kenai & 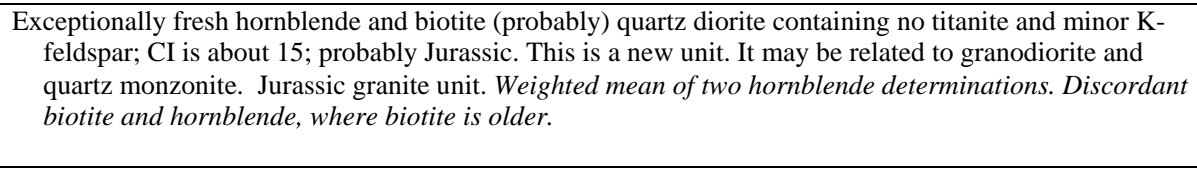 \\
\hline 56 & 77AR 190 & 60.5104 & 153.1793 & Granodiorite & Amphibole & K/Ar & 0.358 & 0.006 & 0.4258 & 51.9 & 81.7 & 2.5 & Lake Clark & 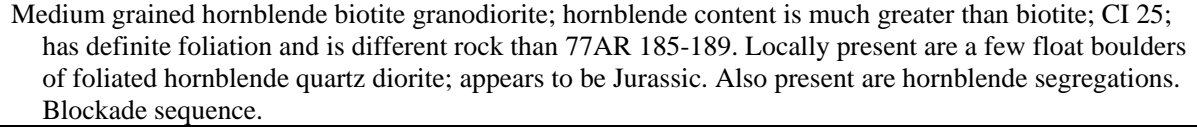 \\
\hline 57 & 78AR 278 & 60.5071 & 153.5526 & Granodiorite & $\frac{\text { Biotite }}{\text { Horblende }}$ & K/Ar & $\frac{9.030}{0.78}$ & 0.014 & $\frac{9.765}{0.0832}$ & $\frac{75.9}{70.7}$ & $\frac{73.6}{85.5}$ & $\frac{2.2}{2.6}$ & Lake Clark & $\begin{array}{l}\text { Hornblende biotite granodioritite containing abundant titanitie. This is same rocks as } 78 \text { AR } 276 \text {. Second } \\
\text { quartz monzodiorite unit. Discordant biotite and horrblende. }\end{array}$ \\
\hline 58 & 81AR 173 & 60.5065 & 152.5646 & Quartz monzonite & $\begin{array}{l}\frac{\text { Biotite }}{\text { Hornblende }} \\
\end{array}$ & K/Ar & $\begin{array}{l}7.505 \\
0.678\end{array}$ & $\begin{array}{l}0.049 \\
0.001\end{array}$ & $\frac{19.90}{1.738}$ & $\begin{array}{l}88.6 \\
87.7\end{array}$ & $\begin{array}{l}175.4 \\
170\end{array}$ & $\begin{array}{l}5.3 \\
5.1\end{array}$ & Kenai & 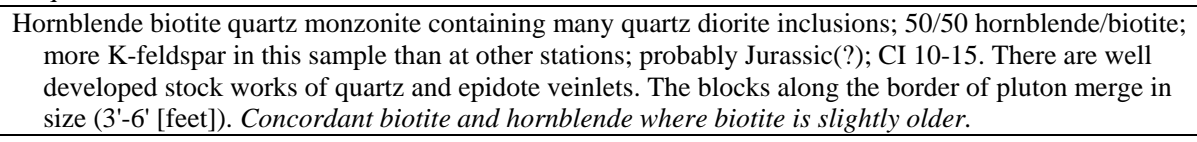 \\
\hline 59 & 78AR 273 & 60.472 & 153.7076 & Granodiorite & $\begin{array}{l}\text { Biotite } \\
\text { Hornblende }\end{array}$ & $\begin{array}{l}\mathrm{K} / \mathrm{Ar} \\
\mathrm{K} / \mathrm{Ar}\end{array}$ & $\begin{array}{l}8.885 \\
0.536\end{array}$ & $\begin{array}{l}0.007 \\
0.006\end{array}$ & $\begin{array}{l}4.928 \\
0.2827\end{array}$ & $\begin{array}{l}36.8 \\
53.8\end{array}$ & $\begin{array}{l}38.1 \\
36.3\end{array}$ & $\begin{array}{l}1.1 \\
1.1\end{array}$ & Lake Clark & $\begin{array}{l}\text { Medium grained biotite and hornhlende granodiorite to quartz diorite not containing titanite and having } \\
\text { accessory inclusions 3-5 inches across; Cl is approximately 12. Concordant biotite and hornblende } \\
\text { where biotite is slightly older. }\end{array}$ \\
\hline 60 & 81AR 178 & 60.4702 & 152.8994 & Granodiorite & Biotite & K/Ar & 9.015 & 0.022 & 21.08 & 93.0 & 156 & 4.7 & Kenai & 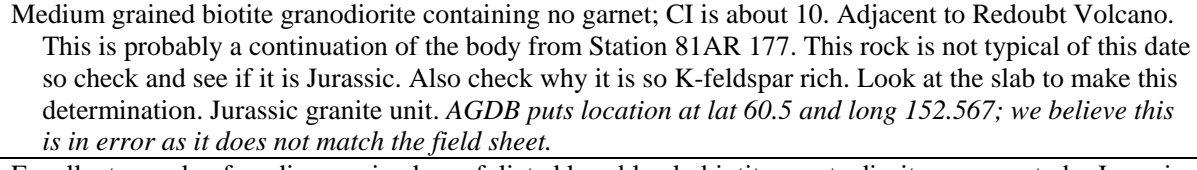 \\
\hline 61 & 72AR 44 & 60.4563 & 153.1439 & Quartz diorite & $\begin{array}{l}\text { Biotite } \\
\text { Hornblende }\end{array}$ & $\begin{array}{l}\mathrm{K} / \mathrm{Ar} \\
\mathrm{K} / \mathrm{Ar}\end{array}$ & $\begin{array}{l}9.165 \\
0.427\end{array}$ & 0.021 & $\frac{9.342}{0.4763}$ & $\begin{array}{ll}77.8 \\
27.2\end{array}$ & 69.5 & 2.1 & Lake Clark & $\begin{array}{l}\text { Excellent sample of medium grained non foliated hormblende-biotite quartz diorite; appears to be Jurassic. } \\
\text { Blockade sequence. Recalculatated with constants of Steiger and Jager (1977). Discordant biotite and } \\
\text { hornblende. }\end{array}$ \\
\hline 62 & 78AR 123 & 60.4509 & 153.513 & Granodiorite & Biotite & K/Ar & 9.105 & 0.022 & 5.495 & 73.1 & 41.4 & 1.2 & Lake Clark & 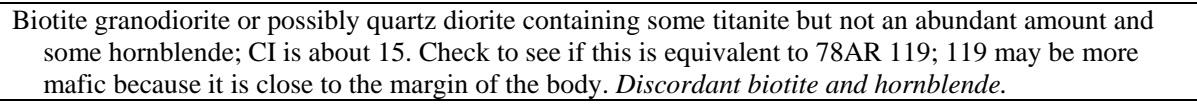 \\
\hline 63 & 77AR 189 & $\begin{array}{ll}60.4408 \\
\end{array}$ & 153.183 & $\begin{array}{l}\text { Quartz diorite } \\
\end{array}$ & $\begin{array}{l}\text { Biotite } \\
\text { Hormblende }\end{array}$ & $\begin{array}{l}\mathrm{K} / \mathrm{Ar} \\
\mathrm{K} / \mathrm{Ar}\end{array}$ & $\begin{array}{l}9.135 \\
0.436\end{array}$ & $\begin{array}{l}0.008 \\
0.003\end{array}$ & $\begin{array}{l}9.926 \\
0.4877\end{array}$ & $\begin{array}{l}84.2 \\
30.5\end{array}$ & 73.9 & $\frac{2.2}{2.3}$ & Lake Clark & 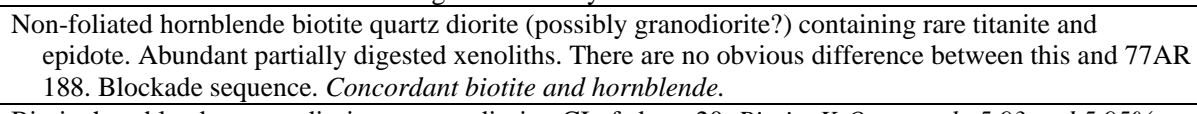 \\
\hline 64 & 72AR 290 & 60.4406 & $\begin{array}{l}153.562 \\
\end{array}$ & Quartz diorite & Hornblende & $\begin{array}{c}\text { K/Ar } \\
\begin{array}{c}\text { weighted } \\
\text { mean }\end{array} \\
\mathrm{K} / \mathrm{Ar} \\
\begin{array}{l}\text { weighted } \\
\text { mean }\end{array}\end{array}$ & 5.94 & $\begin{array}{ll}0.014 \\
\end{array}$ & $\begin{array}{l}4.819 \\
4.802\end{array}$ & $\begin{array}{l}83.0 \\
86.1\end{array}$ & $\begin{array}{l}55.5 \\
55.3 \\
55.4 \\
34.7 \\
36.4\end{array}$ & $\begin{array}{l}1.7 \\
1.7 \\
\mathbf{1 . 2} \\
1.0 \\
1.1\end{array}$ & Lake Clark & 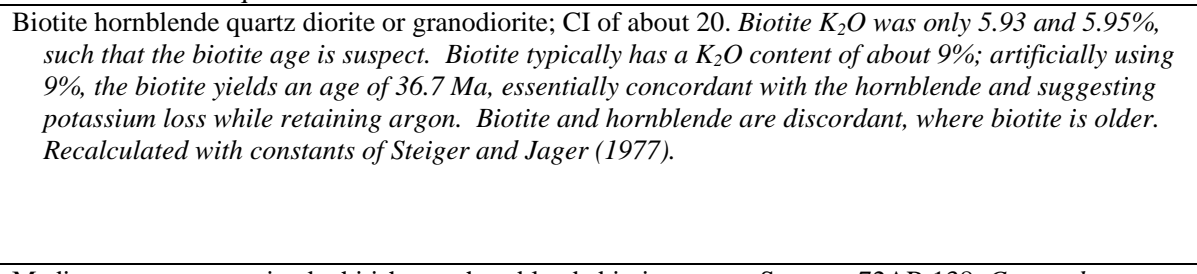 \\
\hline 65 & 72AR 139 & 60.4267 & 153.3375 & Granodiorite & $\begin{array}{l}\text { Biotite } \\
\text { Hornblende }\end{array}$ & $\begin{array}{l}\mathrm{K} / \mathrm{Ar} \\
\mathrm{K} / \mathrm{Ar} \\
\begin{array}{l}\text { weighted } \\
\text { mean }\end{array} \\
\end{array}$ & $\begin{array}{l}8.960 \\
0.943\end{array}$ & $\begin{array}{l}0.014 \\
0.004\end{array}$ & $\begin{array}{l}9.962 \\
1.084 \\
1.069 \\
1.021\end{array}$ & $\begin{array}{l}69.2 \\
76.3 \\
72.2 \\
74.6\end{array}$ & $\begin{array}{l}75.7 \\
78.2 \\
77.0 \\
73.7 \\
76.2 \\
\end{array}$ & $\begin{array}{l}2.3 \\
2.3 \\
2.3 \\
2.2 \\
1.3 \\
\end{array}$ & Lake Clark & $\begin{array}{l}\text { Medium- to coarse-grained whitish gray hornblende biotite quartz. Same as } 72 \text { AR } 138 \text {. Concordant } \\
\text { biotite and hornblende. Blockade sequence. Recalculated with constants of Steiger and Jager (1977). }\end{array}$ \\
\hline 66 & 72AR 138 & 60.3985 & 153.4448 & Quartz diorite & $\begin{array}{l}\text { Biotite } \\
\text { Hornblende }\end{array}$ & K/Ar & $\begin{array}{l}8.950 \\
0.990\end{array}$ & 0.028 & $\frac{8.862}{1.189}$ & 84.7 & $\frac{67.5}{81.6}$ & $\frac{2}{2.4}$ & Lake Clark & 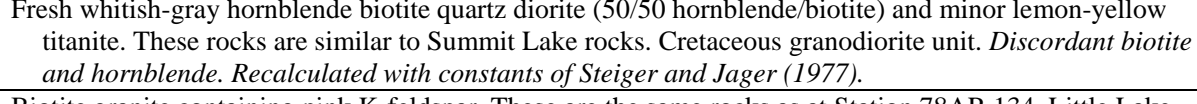 \\
\hline 67 & 78AR 137 & 60.3908 & 153.6782 & Granodiorite & Biotite & K/Ar & 8.625 & 0.007 & 4.661 & 68.6 & 37.1 & 1.1 & Lake Clark & $\begin{array}{l}\text { Biotite granitit containing pink K-feldspar. These are the same rocks as at Station 78AR 134. Little Lake } \\
\text { Clark biotite granite unit. }\end{array}$ \\
\hline 68 & 78AR 156 & 60.3854 & 153.0904 & Quartz diorite & $\frac{\text { Biotite }}{\text { Hornblende }}$ & K/Ar & $\frac{8.655}{0.895}$ & 0.007 & $\frac{18.99}{2.119}$ & $\begin{array}{lll}94.9 \\
37.9\end{array}$ & 146 & $\begin{array}{ll}4.4 \\
4.7\end{array}$ & Lake Clark & $\begin{array}{l}\text { Hornblende biotitie quartz diorite; typical Jurassic; CI 25; little or no K-feldspar. Unit is Jurassic tonalite. } \\
\text { Discordant biotite and horblende. }\end{array}$ \\
\hline 69 & 77AR 186 & 60.3452 & 153.3684 & Quartz diorite & $\begin{array}{l}\frac{\text { Biotite }}{\text { Hormblende }} \\
\end{array}$ & K/Ar & $\frac{9.76}{0.918}$ & 0.028 & $\frac{9.637}{0.9261}$ & $\frac{62.2}{35.4}$ & 67.6 & $\frac{2.0}{2.1}$ & Lake Clark & $\begin{array}{l}\text { Coarse grained biotite hornblende quartz diorite containing } 1 \% \text { titanite and } 2-3 \% \text { epidote; good foliation; } \\
\text { CI II5-20; same rock type as 77AR } 185 \text {. Cretaceous granodionite unit. Concordant biotitie and } \\
\text { hornblende. }\end{array}$ \\
\hline 70 & 72AR 278 & 60.3422 & 153.7964 & Granodiorite & Biotite & K/Ar & 8.26 & 0.015 & 5.328 & 74.4 & 44.3 & 1.3 & Lake Clark & $\begin{array}{l}\text { Orange bioitite uuartz monzonite or quartz dioritit containing minor hornblende. Tazamina granite. } \\
\text { Recalcultated with constants of Steiger and Jager (1977). }\end{array}$ \\
\hline 71 & 72AR 174 & 60.341 & 153.599 & Quartz diorite & Hornblende & K/Ar & 0.532 & 0.011 & 0.2692 & 55.3 & 35.1 & 1.1 & Lake Clark & $\begin{array}{l}\text { Medium-whititsh gray non--oliated biotite horrbblende quartz dioritite with reddish non-foliated diogitic } \\
\text { inclusion. These might be Summit Lake rocks. Recalculated with constants of Steiger and Jager } \\
\text { (1977). }\end{array}$ \\
\hline 72 & 72AR 172 & 60.3285 & 153.5055 & Granodiorite & $\begin{array}{ll}\text { Hornblende } \\
\text { Biotite }\end{array}$ & $\begin{array}{ll}\mathrm{K} / \mathrm{Ar} \\
\mathrm{K} / \mathrm{Ar}\end{array}$ & $\frac{0.957}{9.230}$ & $\begin{array}{ll}0.0 \\
0.0\end{array}$ & $\frac{1.144}{9.262}$ & $\begin{array}{ll}69.2 \\
80.0\end{array}$ & $\frac{81.2}{68.4}$ & $\frac{2.4}{2.1}$ & Lake Clark & $\begin{array}{l}\text { Medium- to fine-grained biotite quartz dionite to granodiorite and possibly may include some horblende. } \\
\text { Cretaceous granodiorite unit. Discordant biotite and hornblende. Recalculatede with constants of } \\
\text { Steiger and Jager (1977). }\end{array}$ \\
\hline 73 & 72AR 136 & 60.3253 & 153.6527 & Granodiorite & $\begin{array}{l}\text { Biotite } \\
\text { Hornblende }\end{array}$ & K/Ar & $\begin{array}{l}8.870 \\
0.857\end{array}$ & 0.028 & $\begin{array}{l}4.506 \\
0.5368\end{array}$ & $\begin{array}{l}80.0 \\
52.1\end{array}$ & 34.99 & $\begin{array}{l}1.0 \\
1.3\end{array}$ & Lake Clark & 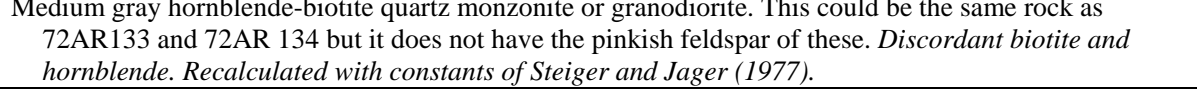 \\
\hline 74 & 77AR 175 & 60.3233 & 153.8505 & Quartz diorite & $\begin{array}{l}\text { Biotite } \\
\text { Hornblende }\end{array}$ & K/Ar & $\begin{array}{l}7.505 \\
0.662\end{array}$ & $\frac{0.007}{0.003}$ & $\frac{6.153}{0.5696}$ & $\begin{array}{l}42.1 \\
70.8\end{array}$ & $\frac{56.1}{58.8}$ & $\frac{1.7}{1.8}$ & Lake Clark & 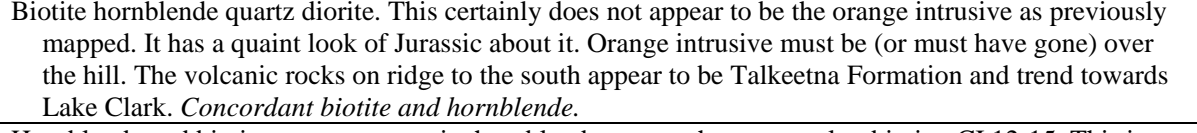 \\
\hline 75 & 77AR 179 & 60.3155 & 1533.8117 & Quartz monzonite & $\begin{array}{l}\text { Biotite } \\
\text { Hornblende }\end{array}$ & $\frac{\mathrm{K} / \mathrm{Ar}}{\mathrm{K} / \mathrm{Ar}}$ & $\begin{array}{l}8.125 \\
0.526\end{array}$ & 0.035 & $\begin{array}{l}4.109 \\
0.2834\end{array}$ & $\begin{array}{l}59.6 \\
41.3\end{array}$ & $\begin{array}{l}34.8 \\
37.0\end{array}$ & $\begin{array}{l}1.0 \\
1.1\end{array}$ & La & 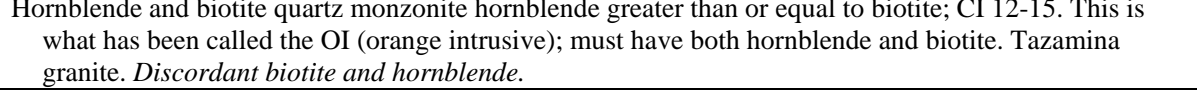 \\
\hline
\end{tabular}




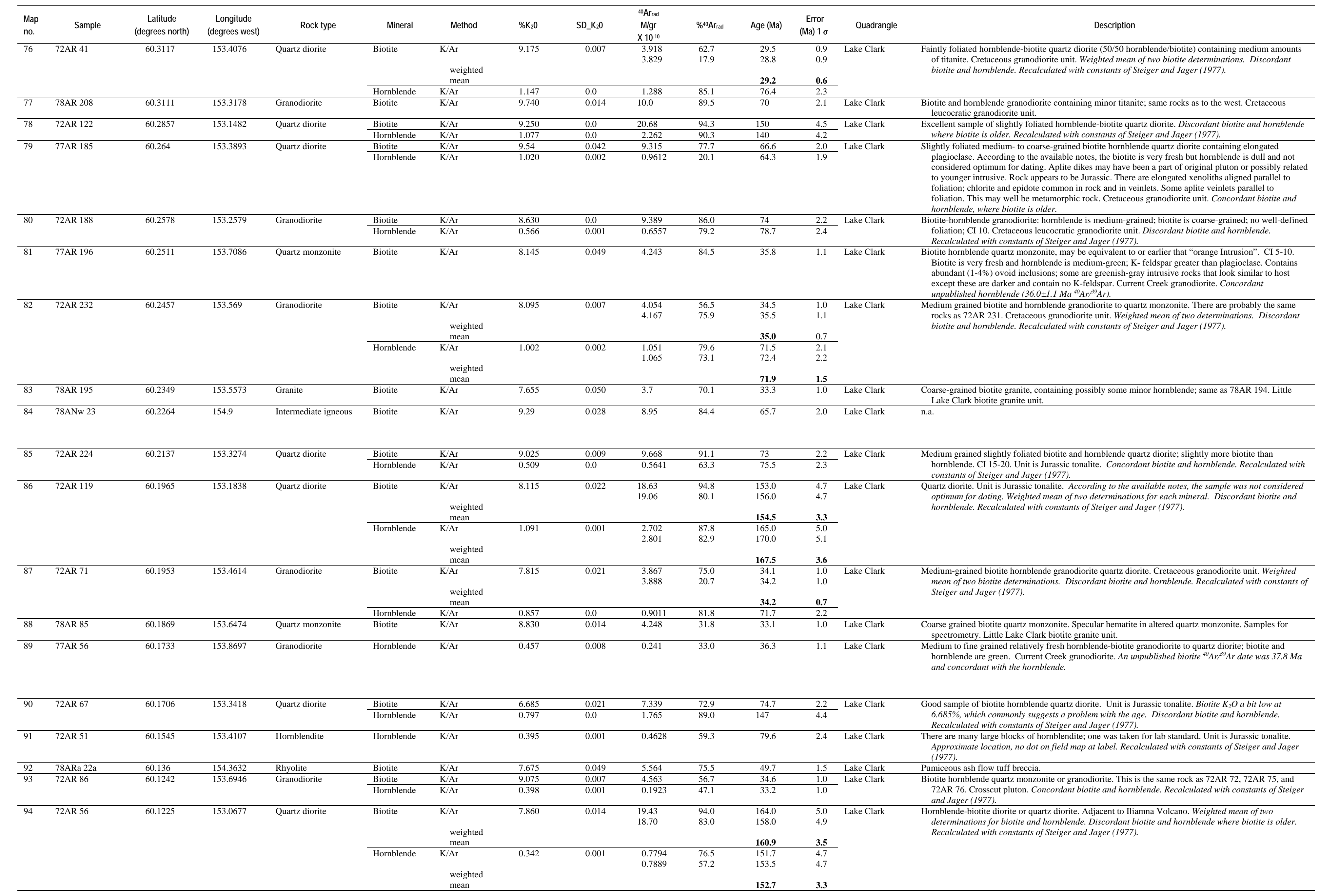




\begin{tabular}{|c|c|c|c|c|c|c|c|c|c|c|c|c|c|}
\hline $\begin{array}{l}\text { Map } \\
\text { no. }\end{array}$ & Sample & $\begin{array}{c}\text { Latitude } \\
\text { (degrees north) }\end{array}$ & $\begin{array}{l}\text { Longitude } \\
\text { (degrees west) }\end{array}$ & Rock type & Mineral & Method & $\% \mathrm{~K}_{2} \mathrm{O}$ & SD_K $K_{2} \mathrm{O}$ & 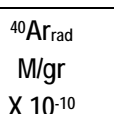 & $\%^{* 4} \mathrm{Ar}_{\mathrm{rad}}$ & Age (Ma) & $\begin{array}{l}\text { Error } \\
\text { (Ma) } 1 \sigma\end{array}$ & Quadrangle \\
\hline 95 & 77AR 60 & 60.1141 & 153.5375 & Granodiorite & $\begin{array}{l}\frac{\text { Biotite }}{\text { Hornblende }} \\
\end{array}$ & $\begin{array}{l}\mathrm{K} / \mathrm{Ar} \\
\mathrm{K} / \mathrm{Ar} \\
\begin{array}{l}\text { weighted } \\
\text { mean }\end{array}\end{array}$ & $\begin{array}{l}9.045 \\
0.472\end{array}$ & $\begin{array}{l}0.035 \\
0.012\end{array}$ & $\begin{array}{l}4.243 \\
0.1758 \\
0.2053\end{array}$ & $\begin{array}{l}57.2 \\
14.5 \\
31.5\end{array}$ & $\begin{array}{l}32.3 \\
25.7 \\
30.0 \\
27.6\end{array}$ & $\begin{array}{l}1 \\
0.8 \\
0.9 \\
\mathbf{0 . 6}\end{array}$ & Lake Clark \\
\hline 96 & 77AR 48 & 60.0979 & 153.5565 & Quartz monzonite & Biotite & K/Ar & 9.505 & 0.007 & 4.369 & 60.3 & 31.7 & 1.0 & Lake Clark \\
\hline 97 & $72 \mathrm{AR} 76$ & 60.0881 & 153.7206 & Granodiorite & $\begin{array}{l}\text { Biotite } \\
\text { Hornblende }\end{array}$ & $\begin{array}{l}\mathrm{K} / \mathrm{Ar} \\
\mathrm{K} / \mathrm{Ar}\end{array}$ & $\begin{array}{l}8.715 \\
0.537\end{array}$ & $\begin{array}{l}0.021 \\
0.004\end{array}$ & $\begin{array}{l}4.343 \\
0.2345\end{array}$ & $\begin{array}{r}77.5 \\
33.7\end{array}$ & $\begin{array}{l}34.2 \\
30.1\end{array}$ & $\begin{array}{l}1.0 \\
0.9\end{array}$ & Lake Clark \\
\hline 98 & 77AR 84 & 60.0704 & 153.9692 & Granodiorite & $\begin{array}{l}\frac{\text { Biotite }}{\text { Hornblende }} \\
\end{array}$ & $\begin{array}{l}\mathrm{K} / \mathrm{Ar} \\
\mathrm{K} / \mathrm{Ar} \\
\begin{array}{l}\text { weighted } \\
\text { mean }\end{array}\end{array}$ & $\begin{array}{l}7.055 \\
0.565\end{array}$ & $\begin{array}{l}0.049 \\
0.004\end{array}$ & $\begin{array}{l}4.046 \\
0.2973 \\
0.2854\end{array}$ & $\begin{array}{l}27.1 \\
29.2 \\
19.1\end{array}$ & $\begin{array}{l}39.4 \\
36.2 \\
34.8\end{array}$ & $\begin{array}{l}.2 \\
1.1 \\
1.0\end{array}$ & Lake Clark \\
\hline
\end{tabular}

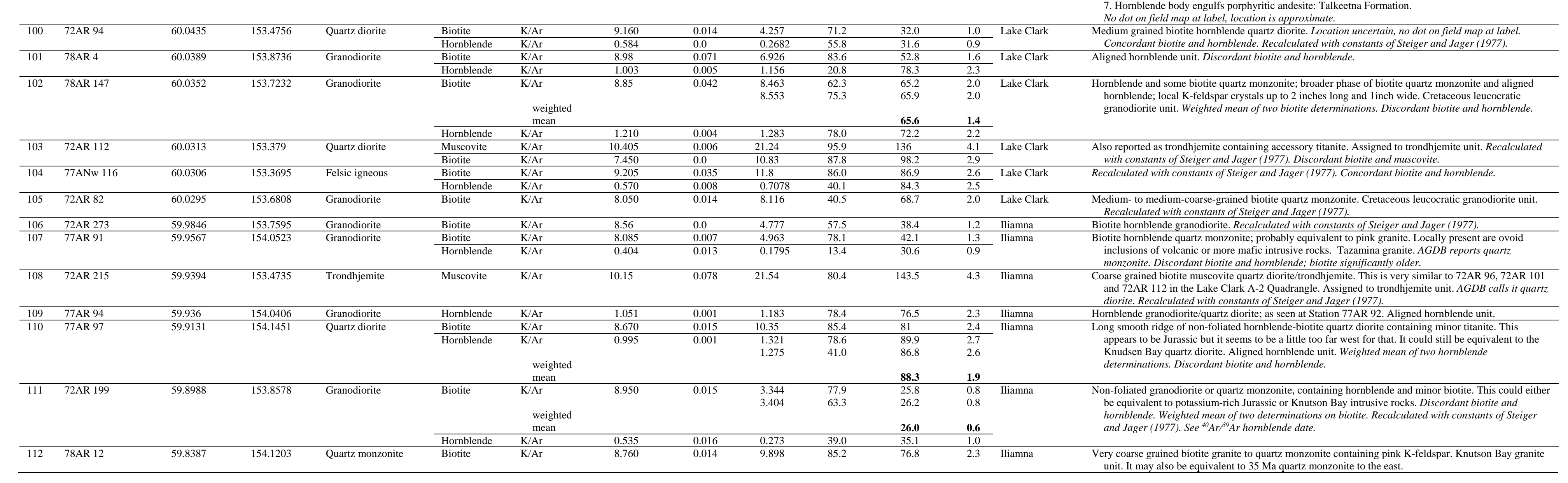


Table 2. Descriptive information and ${ }^{40} \mathrm{Ar} / 39 \mathrm{Ar}$ analytical data for samples of the Alaska-Aleutian Range batholith in the Lime Hills 1:250,000-scale quadrangles, south-central Alaska.

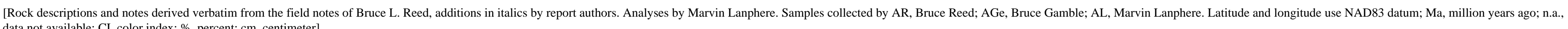
data not available; CI, color index; \%, percent; cm, centimeter!

\begin{tabular}{|c|c|c|c|c|c|c|c|c|c|c|c|c|c|c|c|c|}
\hline $\begin{array}{l}\text { Map } \\
\text { letter }\end{array}$ & Sample & $\begin{array}{l}\text { Latitude } \\
\text { (degrees north) }\end{array}$ & $\begin{array}{c}\text { Longitude } \\
\text { (degrees west) }\end{array}$ & Rock type & Mineral & ${ }^{40 \mathrm{Ar} / 3 \mathrm{Ar}}$ & ${ }^{37 \mathrm{Ar} P \mathrm{PAr}}$ & ${ }^{36 \mathrm{Ar} P \mathrm{~B} A \mathrm{Ar}}$ & 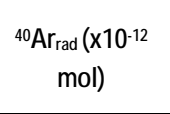 & ${ }^{\%{ }^{*} \mathrm{Ar} \text { frad }}$ & KICa & $\mathrm{J}$ & Age (Ma) & $\begin{array}{l}\text { Error } \\
\text { (Ma) } \\
1 \sigma\end{array}$ & Quadrangle & Description \\
\hline $\begin{array}{ll}\mathrm{A} \\
\mathrm{B}\end{array}$ & $\begin{array}{l}88 \mathrm{AR} 225 \\
88 \mathrm{AR} 238\end{array}$ & 61.9917 & $\begin{array}{l}153.5431 \\
154.3584\end{array}$ & $\begin{array}{c}\text { Granodiorite } \\
\text { Granodiorite }\end{array}$ & $\begin{array}{c}\text { Biotite } \\
\text { Biotite }\end{array}$ & $\begin{array}{ll}3.391 \\
4.323\end{array}$ & $\begin{array}{l}0.02071 \\
0.03556\end{array}$ & $\begin{array}{l}0.003564 \\
0.003356\end{array}$ & $\begin{array}{c}9.249 \\
64.47\end{array}$ & $\begin{array}{ll}69.0 \\
77.1\end{array}$ & 13.8 & $\begin{array}{ll}0.0110 \\
0.01082\end{array}$ & $\begin{array}{ll}45.8 \\
63.9\end{array}$ & $\begin{array}{l}0.7 \\
0.5\end{array}$ & $\begin{array}{l}\text { Lime Hills } \\
\text { Lime Hills }\end{array}$ & 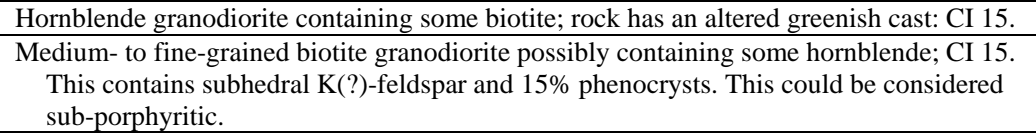 \\
\hline $\mathrm{C}$ & 88AR 241 & 61.9044 & 153.235 & Granodiorite & 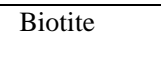 & 3.674 & 0.02482 & 0.005757 & 31.97 & 53.7 & 19.7 & 0.01096 & 38.6 & 0.4 & Lime Hills & 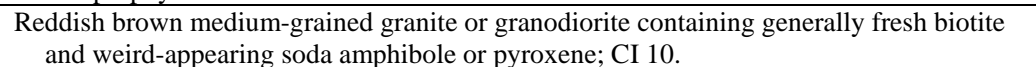 \\
\hline $\mathrm{D}$ & 88AR 220 & 61.8394 & 153.5217 & Granodiorite & Biotite & 7.087 & 0.00928 & 0.001585 & 31.32 & 93.3 & 52.8 & 0.00556 & 65.1 & 0.4 & Lime Hills & 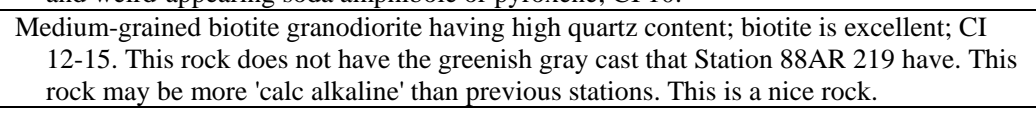 \\
\hline $\mathrm{E}$ & 88AR 219 & 61.8039 & 153.6058 & Granodionite & Hormblende & 4.176 & 0.03417 & 0.002852 & 22.88 & 79.9 & 14.3 & 0.01102 & 65.1 & 0.6 & Lime Hills & $\begin{array}{l}\text { Medium--ine grained biotite granodiorite containing altered clots of what might be } \\
\text { pyyorene CII } 15 .\end{array}$ \\
\hline $\mathrm{F}$ & 88AR 245 & 61.7408 & 153.1908 & Granite & Biotite & 5.075 & 0.06988 & 0.007588 & 52.96 & 55.9 & 7.0 & 0.01112 & 56.0 & 0.5 & Lime Hills & $\begin{array}{l}\text { Very fresh slightly porphyyritic biotite granite containing K-feldspar, quartz, and possibly a a } \\
\text { littl horblende. This sock is probably the coro of the inturion and probably the main } \\
\text { rock type. It appears to be part of the Merrill Pass sequence. }\end{array}$ \\
\hline G & 88AR 246 & 61.6692 & 153.2839 & Granite & Biotite & 3.723 & 0.08465 & 0.005258 & 6.792 & 58.4 & 5.8 & 0.01072 & 41.6 & 0.8 & Lime Hills & 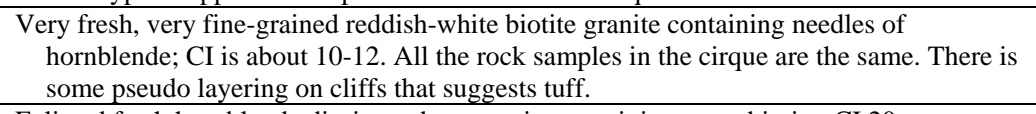 \\
\hline $\mathrm{H}$ & 88AR 234 & 61.5594 & 153.7053 & Monzonite & $\begin{array}{l}\text { Biotite } \\
\text { Hornblende }\end{array}$ & $\begin{array}{l}7.720 \\
9.163\end{array}$ & 0.04859 & $\begin{array}{ll}0.001746 \\
0.0049975\end{array}$ & $\frac{30.98}{2.546}$ & $\begin{array}{l}93.3 \\
98.3\end{array}$ & $\frac{10.1}{0.12}$ & 0.005505 & 70.1 & $\begin{array}{c}0.4 \\
0.5\end{array}$ & Lime Hills & $\begin{array}{l}\text { Foliated fresh horblenlende diorite and monzonite containing some biotite: CI } 20 . \\
\text { Discordant biotite and hornblende. }\end{array}$ \\
\hline I & 88AR 266 & 61.5342 & 153.6767 & Monzonite & Hornblende & 6.902 & 5.257 & 0.01268 & 1.483 & 51.7 & 0.10 & 0.01162 & 73.5 & 5.3 & Lime Hills & $\begin{array}{l}\text { Hornblende monzodiorite to diorite containing some fresh biotite; both hornblende and } \\
\text { biotite are very good; CI } 20 \text {. }\end{array}$ \\
\hline J & 88AR 268C & 61.4867 & 153.6894 & $\begin{array}{l}\text { Tuff } \\
\end{array}$ & Hornblende & 7.44 & 8.203 & 0.02134 & 1.205 & 23.8 & 0.10 & 0.01053 & 33.6 & 3.4 & $\begin{array}{c}\text { Lime Hills } \\
\text { L }\end{array}$ & $\begin{array}{l}\text { Coarse grained greenish crystal-rich inclusion-rich vent(?) material containing biotite and } \\
\text { possibly some horrblende, inclusion clasts of } 76 \text { Ma intrusive up to } 1 \text { i inches?. Therere } \\
\text { are two granitic types in this rock no inclusions of flysch (Kahiltna). }\end{array}$ \\
\hline $\mathrm{K}$ & 88AR 256 & 61.4469 & 153.5819 & Monzodiorite & Biotite & 3.984 & 0.0201 & 0.00242 & 32.2 & 82.1 & 24.4 & 0.01118 & 64.8 & 0.5 & Lime Hills & $\begin{array}{l}\text { Some altered biotite hornblende monzodiorite; the biotite is fine-grained and reddish } \\
\text { brown and the rock itself is ok; forget about the hornblende; CI 15. }\end{array}$ \\
\hline $\mathrm{L}$ & 88AR 216 & 61.4353 & 153.815 & $\begin{array}{l}\text { Granodiorite } \\
\text { G }\end{array}$ & 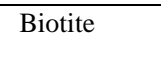 & 2.601 & 0.01079 & 0.002733 & 27.16 & 68.9 & 45.4 & 0.01121 & 35.9 & 0.3 & Lime Hills & 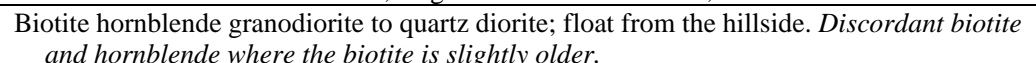 \\
\hline $\mathrm{M}$ & 87AR 38 & 61.4261 & 153.395 & $\begin{array}{l}\text { Granodiorite } \\
\end{array}$ & 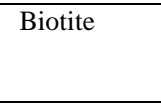 & 5.414 & 0.09572 & 0.005796 & 9.808 & 68.4 & 5.1 & 0.00552 & 36.5 & 0.3 & Lime Hills & $\begin{array}{l}\text { Intrusiver rock containing biotite and minor amphibole(?). This is not a lithic tuff as } \\
\text { thought. II cuuld be a late stage dome-type intrusive. The biotite should provide good } \\
\text { clues as to the minimum age of the volcanic rocks. }\end{array}$ \\
\hline$\frac{\mathrm{N}}{\mathrm{O}}$ & $\frac{91 \mathrm{AL} 8}{88 \mathrm{AR} 249}$ & $\frac{61.4236}{61.3639}$ & $\begin{array}{l}153.1095 \\
153.4767\end{array}$ & $\begin{array}{l}\text { Granodiorite } \\
\text { Rhyolite }\end{array}$ & $\begin{array}{c}\text { Biotite } \\
\text { Biotite }\end{array}$ & 3.293 & $\begin{array}{l}0.1614 \\
0.2813\end{array}$ & $\begin{array}{l}0.004313 \\
0.008216\end{array}$ & 26.94 & $\frac{61.5}{46.3}$ & $\begin{array}{l}3.0 \\
1.7\end{array}$ & 0.01119 & 40.4 & $\begin{array}{c}5.2 \\
1.6\end{array}$ & $\begin{array}{l}\text { Lime Hills } \\
\text { Lime Hills }\end{array}$ & $\begin{array}{l}\text { n.a. } \\
\text { Fresh columnar jointed biotite-bearing rhyolite ash flow. The sanidine is not as good as } \\
\text { the biotite. } 0 \text { som sanidine has fresc hleavage faces, tuff includes quartz phenocrysts. } \\
\text { The high peak in the north is at least } 300 \text { meterers of ash. }\end{array}$ \\
\hline $\mathrm{P}$ & 88AR 235 & 61.3542 & 153.6711 & Granodionite & Biotite & 2.537 & 0.02028 & 0.00256 & 24.58 & 70.2 & 24.2 & 0.01120 & 35.6 & 0.3 & Lime Hills & $\begin{array}{l}\text { Medium-grained granodiorite containing good biotite and minor, possibly chloritized, } \\
\text { hornblende; CI i a about } 15\end{array}$ \\
\hline Q & 88AR 261 & 61.3506 & 153.3914 & $\begin{array}{l}\text { Tuff } \\
\end{array}$ & 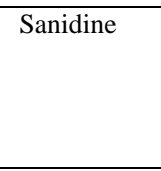 & 4.596 & 0.2927 & 0.009325 & 23.54 & 40.4 & 1.7 & 0.01133 & 37.6 & 1.8 & Lime Hills & 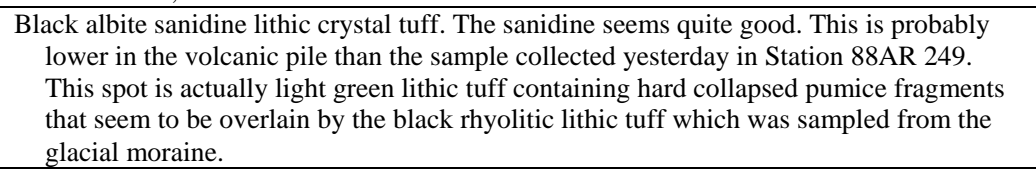 \\
\hline $\mathrm{R}$ & 87AR 61 & 61.2689 & 153.4733 & Granite & Biotite & 4.246 & 0.03967 & 0.000656 & 2.78 & 95.4 & 12.4 & 0.00578 & 42.2 & 0.3 & Lime Hills & $\begin{array}{l}\text { Biotite granite containing a trace of hornblende. This is what we have been calling the } \\
\text { Merrifi Pass sequence. The only thing that differs in the Merrill Pass rocks is the } \\
\text { amount of hornblende but the difference is not enough to worly about at the moment. }\end{array}$ \\
\hline $\mathrm{s}$ & 87AGe 37 & 61.2111 & 153.6158 & Granodiorite & 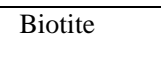 & 4.333 & 0.04146 & 0.00210 & 17.4 & 85.6 & 11.8 & 0.00578 & 38.3 & 0.3 & Lime Hills & 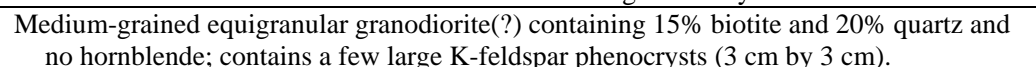 \\
\hline $\mathrm{T}$ & 87AGe 44 & 61.0189 & 153.6481 & Granodiorite & Hornblende & 10.481 & 10.65 & 0.01688 & 7.478 & 60.3 & 0.046 & 0.00544 & 61.4 & 0.7 & Lime Hills & $\begin{array}{l}\text { Medium-grained equibranaluarar granodidiorite containing about 15\% hornblende and 15-20\% } \\
\text { quartz and no biotite like other stations in this intusive. }\end{array}$ \\
\hline E & 88AR 219 & 61.8039 & 153.6058 & Granodiorite & Hornblende & 4.176 & 0.03417 & 0.002852 & 22.88 & 79.9 & 14.3 & 0.01102 & 65.1 & 0.6 & Lime Hills & $\begin{array}{l}\text { Medium-fine grained bioite granodiorite containing altered clots of what might be } \\
\text { pyroxen; } \mathrm{CI} 15 \text {. }\end{array}$ \\
\hline $\mathrm{F}$ & 88AR 245 & 61.7408 & 153.1908 & Granite & Biotite & 5.075 & 0.06988 & 0.007588 & 52.96 & 55.9 & 7.0 & 0.01112 & 56.0 & 0.5 & Lime Hills & $\begin{array}{l}\text { Very fresh slightly porphyritic biotite granite containing K-feldspar, quartz, and possibly a a } \\
\text { littl horblende. This sork is probably the coro of the inturion and probably the main } \\
\text { rock type. It appears to be part of the Merill Pass sequence. }\end{array}$ \\
\hline G & 88AR 246 & 61.6692 & 153.2839 & Granite & Biotite & 3.723 & 0.08465 & 0.005258 & 6.792 & 58.4 & 5.8 & 0.01072 & 41.6 & 0.8 & Lime Hills & $\begin{array}{l}\text { Very fresh, very fine-grained reddish-white biotite granite containing needles of } \\
\text { hornblende; CI I i about 10-12. All the rock samples in the cirque are the same. There is } \\
\text { some pseudo layering on cliffs that suggests tuff. }\end{array}$ \\
\hline $\mathrm{H}$ & 88AR 234 & 61.5594 & 153.7053 & $\begin{array}{l}\text { Monzonite } \\
\text {. }\end{array}$ & $\begin{array}{ll}\text { Biotite } \\
\text { Horble }\end{array}$ & $\frac{7.720}{993}$ & 0.04859 & $\begin{array}{ll}0.001746 \\
0.009995\end{array}$ & $\frac{30.98}{2.54}$ & $\frac{93.3}{98.3}$ & $\frac{10.1}{0.12}$ & 0.005505 & 70.1 & $\frac{0.4}{0.5}$ & Lime Hills & $\begin{array}{l}\text { Foliated fresh hornblende diorite and monzonite containing some biotite: } \mathrm{CI} 20 . \\
\text { Discordant bibitita and horblende. }\end{array}$ \\
\hline I & 88AR 266 & 61.5342 & 153.6767 & Monzonite & Hornblende & 6.902 & 5.25 & 0.01268 & 1.483 & 51. & 0.10 & 0.01162 & 73.5 & 5.3 & Lime Hills & $\begin{array}{l}\text { Hornblende monzodiorite to diorite containing some fresh biotite; both hornblende and } \\
\text { biotite are very good; CI } 20 \text {. }\end{array}$ \\
\hline $\mathrm{J}$ & 88AR 268C & 61.4867 & 153.6894 & Tuff & Hornblende & 7.44 & 8.203 & 0.02134 & 1.205 & 23.8 & 0.10 & 0.01053 & 33.6 & 3.4 & Lime Hills & $\begin{array}{l}\text { Coarse grained greenish crystal-rich inclusion-rich vent(?) material containing biotite and } \\
\text { possibly some horrblende, inclusion clasts of } 76 \text { Ma intrusive up to } 10 \text { inches?. There } \\
\text { are two granitic types in this rock no inclusions of flysch (Kahiltna). }\end{array}$ \\
\hline $\mathrm{K}$ & 88AR 256 & 61.4469 & 153.5819 & $\begin{array}{l}\text { Monzodiorite } \\
-\end{array}$ & 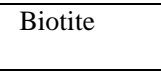 & 3.984 & 0.0201 & 0.00242 & 32.2 & 82.1 & 24.4 & 0.01118 & 64.8 & 0.5 & $\begin{array}{l}\text { Lime Hills } \\
\text { Lits }\end{array}$ & $\begin{array}{l}\text { Some altered biotite horblende monzodiorite; the biotite is fine-grained and reddish } \\
\text { brown and the rock itself is ok; forget about the hornblende; CI } 15 .\end{array}$ \\
\hline $\mathrm{L}$ & 88AR 216 & 61.4353 & 153.815 & Granodiorite & $\frac{\text { Biotite }}{\text { Horblende }}$ & 2.8601 & $\frac{0.01079}{9.007}$ & $\frac{0.002733}{0.02587}$ & $\frac{27.16}{4.913}$ & 68.9 & $\frac{45.4}{0.054}$ & 0.011112 & $\frac{35.9}{34.0}$ & $\frac{0.3}{1.2}$ & Lime Hills & $\begin{array}{l}\text { Biotitit hormblende granodiorite to quartz dioriti, fllat from the hillside. Discordant biotite } \\
\text { and hornblende where the biotitie is slightly older. }\end{array}$ \\
\hline
\end{tabular}




\section{Acknowledgments}

We would like to thank Keith Labay for preparing plate 1 for this report and Nora Shew for originally discovering the existence of the data. We thank R.G. McGimsey, Erin Todd, and J.V. Jones for their thoughtful reviews.

\section{References Cited}

Faure, Gunter, and Mensing, T.M., 2005, Isotopes, principles and applications (3d ed.): Hoboken, NJ, John Wiley and Sons, 897 p.

Granitto, Matthew, Bailey, E.A., Schmidt, J.M., Shew, N.B., Gamble, B.M., and Labay, K.A., 2011, Alaska Geochemical Database (AGDB)—Geochemical data for rock, sediment, soil, mineral, and concentrate sample media: U.S. Geological Survey Data Series 637, 31 p. pamphlet and database, 1 DVD, at http://pubs.usgs.gov/ds/637.

Reed, B.L., and Lanphere, M.A., 1969, Age and chemistry of Mesozoic and Tertiary plutonic rocks in south-central Alaska: Geological Society of America Bulletin, v. 80, p. 23-44.

Reed, B.L., and Lanphere, M.A., 1972, Generalized geologic map of the Alaska-Aleutian Range batholith showing K/Ar ages of the plutonic rocks: U.S. Geological Survey Miscellaneous Field Studies Map MF-372, scale 1:1,000,000.

Reed, B.L., and Lanphere, M.A., 1973, Alaska-Aleutian Range batholith-Geochronology, chemistry and relation of circum-Pacific plutonism: Geological Society of America Bulletin, v. 84, no. 8, p. 2583-2610.

Steiger, R.H., and Jager, E., 1977, Subcommission on geochronology-Convention on the use of decay constants in geo- and cosmochronology: Earth and Planetary Science Letters, v. 36, p. 359-362.

Wilson, F.H., Hults, C.P., Mull, C.G., and Karl, S.M., comps., 2015, Geologic map of Alaska: U.S. Geological Survey Scientific Investigations Map 3340, 196 p., 2 sheets, scale 1:584,000. [Also available at http://dx.doi.org/10.3133/sim3340.] 